\title{
Quels intérêts de la diversité floristique des prairies permanentes pour les ruminants et les produits animaux ?
}

\author{
A. FARRUGGIA ${ }^{1}$, B. MARTIN ${ }^{1}$, R. BAUMONT 1 , S. PRACHE ${ }^{1}$, M. DOREAU ${ }^{1}$, H. HOSTE ${ }^{2}$, D. DURAND ${ }^{1}$ \\ ${ }^{1}$ INRA, UR1213 Herbivores, F-63122 Saint-Genès Champanelle, France \\ 2 INRA, ENV Toulouse, UMR1225, F-31076 Toulouse, France \\ Courriel :farruggi@clermont.inra.fr
}

L'élevage d'herbivores a un impact important sur la biodiversité et en particulier sur celle des prairies permanentes qui sont des réservoirs potentiels de biodiversité végétale. Mais en retour, quels bénéfices les éleveurs peuvent-ils tirer du maintien de cette biodiversité ? Quel est l'intérêt de la diversité floristique des prairies pour la valeur alimentaire des fourrages, la qualité des produits animaux et la santé des animaux ?

Le concept de biodiversité a été défini dans la Convention sur la diversité biologique au Sommet de la Terre de Rio de Janeiro en 1992, comme étant «la variabilité des êtres vivants». Il a été décliné selon trois niveaux d'organisation : la diversité génétique, la diversité des espèces et la diversité des écosystèmes. La biodiversité a été reconnue comme une "préoccupation commune à l'humanité» faisant partie intégrante du processus de développement. Depuis, de nombreux auteurs ont proposé d'aborder la biodiversité selon les fonctions et les services qu'elle remplit, en particulier pour l'agriculture (Swift et al 2004, Clergue et al 2005, Hooper et al 2005, Hector et Bagchi 2007), la notion de service se définissant par les bénéfices qu'en tirent les êtres humains.

L'agriculture, en occupant plus de la moitié du territoire national français, a un rôle central à jouer dans la préservation de la biodiversité surtout si l'on met en parallèle les surfaces bénéficiant d'une protection explicite au titre de la biodiversité qui ne représentent que $12 \%$ du territoire (www. Natura2000.fr). Plus encore, l'élevage d'herbivores, en assurant le maintien et l'entretien des prairies permanentes, considérées comme des réservoirs potentiel de biodiversité animale et végétale et représentant en France environ un tiers de la surface agricole utile (Le Gall et al 2007), occupe une place prépondérante dans la préservation de cette biodiversité. Dans les zones à fortes contraintes naturelles comme les zones de montagne, la plupart des prairies disparaissent sans la présence de l'élevage, c'est-à-dire sans le prélèvement par les animaux ou par la fauche, évoluant vers la friche puis la forêt. L'impact des pratiques agricoles et les liens étroits entre ces pratiques, le milieu physique, la végétation et la faune qui y est inféodée ont été démontrés (Plantureux 1996, Sanderson et al 2007). Par leurs pratiques, les éleveurs influent sur le nombre et les types d'espèces, végétales et animales, présentes dans leurs prairies.

L'objectif de cette synthèse est d'alimenter la problématique des services rendus par la biodiversité à l'élevage, en adoptant un angle de vue principalement zootechnique. Nous n'y abordons qu'une seule des composantes de la biodiversité des prairies permanentes, la diversité floristique. Un état des connaissances est réalisé sur les effets de la composition et de la diversité floristique sur la valeur nutritive des fourrages et sur l'ingestion des animaux, éléments importants des performances animales, sur les caractéristiques nutritionnelles et sensorielles des produits animaux, enfin sur deux aspects de santé animale : la lutte contre les infestations par les strongles digestifs et la prévention des processus de peroxydation. Bien que déterminante pour l'ali- mentation animale, les relations entre la diversité spécifique et la quantité de biomasse fourragère produite ne seront pas évoquées ici. Ce sujet a fait l'objet en effet, de nombreuses publications récentes à caractère plus agronomique qui ont démontré et souligné l'effet positif de l'augmentation de la diversité spécifique sur la production de biomasse aérienne (Hector et al 1999, Sanderson et al 2004, Guo 2007, Kirwan et al 2007, Huyghe et Litrico 2008).

\section{1 / Le rôle des pratiques sur la composition botanique et chimique des prairies per- manentes}

Des travaux récents développés en France et en Suisse ont démontré que la composition botanique et le niveau de diversité floristique (encadré 1) d'une prairie pouvaient être bien expliqués à partir des facteurs du milieu et du mode d'exploitation. La composition botanique résultante influence fortement la composition chimique et en particulier la richesse en composés secondaires.

1.1 / Effets des pratiques et du milieu sur la composition et la diversité floristique

La prairie permanente est constituée d'un mélange important d'espèces 


\section{Encadré 1. Appréciation de la diversité floristique d'une prairie}

La diversité floristique d'une communauté prairiale peut être définie à partir de différentes entités végétales : les espèces ou les groupes d'espèces qu'ils soient fonctionnels comme évoqués dans cet article ou écologiques (regroupement des espèces selon les exigences en eau, lumière, $\mathrm{pH}$...). En supposant la végétation d'une prairie homogène, la diversité moyenne se décline selon les deux critères suivants : la richesse, i.e. le nombre total d'entités végétales (plus le nombre est grand, plus la richesse est élevée) et la régularité de la répartition de ces entités dans la prairie (Loiseau 2004). Ce dernier concept est mathématique et peut être apprécié par exemple à partir de l'indice de Shannon (Magurran 2003). La régularité maximale est obtenue pour une répartition égale des entités en abondance au sein de la prairie.

végétales (de 10 à plus de 100), qui contribuent très diversement à la production de biomasse. Dans beaucoup de situations, la production de biomasse peut être assurée par seulement 2 à 4 espèces dominantes, voire une espèce (Guo 2007). Toutes ces espèces partagent le même habitat et entretiennent des relations de compétition ou de complémentarité pour la lumière, les nutriments et l'eau. Les facteurs de milieu comme l'état hydrique, l'exposition, l'altitude ont des impacts importants sur la composition botanique. Dans la grande majorité des situations agricoles rencontrées en France, ce sont cependant la fertilité du milieu (fertilité intrinsèque du sol et fertilisation) et le taux de défoliation du couvert végétal qui expliquent le mieux la composition et la diversité floristique des prairies (Balent et al 1998, Cruz et al 2002). L'exploitation intense d'une prairie (par ex. fertilisation et chargements élevés) favorise des espèces végétales très compétitives à forte capacité de capture des éléments nutritifs et à vitesse de croissance et de renouvellement des organes élevée. Ces espèces éliminent la plupart des autres et conduisent à une diminution de la richesse spécifique (Duru et al 2004). En revanche, une exploitation très modérée de la prairie, combinant, par exemple une fertilisation et un chargement faibles, favorise les espèces peu compétitives qui présentent des taux de croissance faibles et permet une cohabitation d'un nombre élevé d'espèces ( $>40$ espèces par parcelle). La composition botanique ainsi créée évolue peu au fil d'une année et entre années si les pratiques restent stables, mais la contribution des espèces à la production de biomasse peut varier de façon significative tout au long de la saison. Cette approche de l'évolution de la composition botanique d'une prairie et du nombre d'espèces présentes sous l'effet de la fertilité et de l'utilisation de la parcelle montre $i$ ) qu'il n'est pas possible de concilier, à l'échelle de la parcelle, un niveau de production important et un nombre d'espèces végétales élevé, et ii) que la compréhension de ces évolutions passe par la connaissance des stratégies de vie et des compromis fonctionnels des espèces (Louault et al 2005). A partir d'une approche fonctionnelle de la végétation, il est ainsi possible de regrouper les espèces en groupes dits «fonctionnels» selon les stratégies qu'elles mettent en oeuvre au sein de la communauté prairiale. Grime et al (1988) ont par exemple, classé un nombre très important de plantes en trois grands groupes : les compétitrices (C), les tolérantes au stress $(\mathrm{S})$ et les rudérales (R) qui sont capables de vivre dans des environnements dans lesquels la destruction partielle ou totale de la biomasse est fréquente. Récemment, Cruz et al (2002) et Duru et al (2004) ont utilisé cette approche pour les graminées dont la contribution à la biomasse est généralement très importante et qui constituent de ce fait un marqueur pertinent des caractéristiques agronomiques de la parcelle. Deux grandes stratégies ont été distinguées : l'une correspond à un investissement fort dans la capture des ressources minérales et caractérise les espèces adaptées aux milieux riches et à des fréquences de défoliation élevées (ray-grass anglais, dactyle, pâturin commun), l'autre correspond à la conservation des ressources et caractérise les espèces adaptées à des milieux plus pauvres et à une fréquence de défoliation plus faible (agrostis, fétuque rouge, brachypode penné). Ces deux types de stratégies sont associés à des combinaisons de caractères (appelés aussi «traits») morphologiques (par ex. taille des feuilles, teneur en matière sèche des feuilles (encadré 2)) et physiologiques des espèces (par ex. durée de vie des feuilles, date début floraison). Beaucoup de dicotylédones étant des espèces moins compétitives que les graminées, elles sont bien présentes en terme de nombre d'espèces dans les prairies peu intensément exploitées. Certaines espèces de dicotylédones, comme par exemple le pissenlit, et les légumineuses comme le trèfle blanc et le trèfle violet, classées dans les groupes des espèces compétitrices selon Grime, peuvent néanmoins contribuer à la biomasse de façon relativement importante dans les prairies intensément exploitées.

\section{2 / Les métabolites secondaires présents dans les plantes et leurs fonctions}

Les dicotylédones, nombreuses dans les prairies diversifiées, appartiennent à diverses familles botaniques et ont peu de caractéristiques communes. Cependant, elles sont généralement riches en diverses substances ou métabolites dits secondaires, ce qui les distingue des graminées. Les métabolites secondaires des plantes correspondent aux composés issus des métabolismes qui ne sont associés ni à la nutrition, ni à la croissance des plantes. On attribue généralement à ces métabolites la fonction d'attirer les insectes pollinisateurs, de protéger les plantes dans des conditions de stress et de maintenir leur intégrité contre les compétiteurs, les prédateurs et les agents pathogènes. A titre d'exemples, les tannins sont considérés comme des composés jouant un rôle dans les mécanismes de défense des plantes face à la prédation par des insectes ou des herbivores, et les caroténoïdes interviennent dans la photosynthèse en protégeant la chlorophylle de la chaleur et des radiations solaires. Les composés phénoliques sont les plus nombreux et les plus répandus des métabolites (Wong 1973 cité par Jarrige et al 1995). Il existe ainsi des milliers de molécules ayant une structure polyphénolique (groupement hydroxyl $(\mathrm{OH})$ associé à un cycle aromatique). Chaque espèce contient plusieurs structures phénoliques qui sont souvent spécifiques de l'espèce ou de la famille botanique ou encore des conditions environnementales. Au total, l'ensemble de ces composés représente de 1,5 à $2,5 \%$ de la matière sèche des graminées prairiales des régions tempérées. Leur teneur est plus élevée dans certaines dicotylédones : 3 à $6 \%$ dans le rumex, la bistorte, l'anthrisque (Scehovic 1990 cité par Jarrige et al 1995).

L'influence de la composition botanique reste cependant toujours à relativiser car le stade, les conditions environnementales et le mode de conservation de l'herbe influent fortement sur la concentration de ces différents métabolites.

En conclusion, il est établi que la diversité floristique est une variable «construite» par les pratiques des éleveurs sous la contrainte du milieu. Plus 
le milieu est fertile et la prairie intensément défoliée, plus le nombre d'espèces végétales et en particulier le nombre de dicotylédones est faible. La gamme des composés secondaires est par conséquent plus réduite que celle d'une prairie faiblement utilisée. Cette diversité peut être appréciée à partir du nombre d'espèces ou de groupes d'espèces et de la régularité de leur répartition dans la communauté prairiale (encadré 1). Dans la plupart des références et des thématiques que nous abordons dans cette synthèse, la diversité floristique n'est pas le sujet d'étude et n'est donc pas appréciée en tant que telle. La contribution des familles botaniques (graminées, légumineuses et dicotylédones autres que légumineuses = «diverses») et la richesse en espèces végétales présentes dans la prairie sont les critères le plus fréquemment rencontrés que nous mettons par conséquent le plus en avant. La composition fonctionnelle, basée uniquement sur celle des graminées, est également abordée. Plus rarement, nous avons pu décrire cette diversité par la régularité de la répartition des espèces ou des groupes d'espèces. Il est également apparu nécessaire de s'appuyer sur un deuxième concept, celui de l'identité spécifique i.e. la présence d'une espèce-clé pour une fonction donnée (encadré 1). Enfin, pour plusieurs des thématiques abordées, nous nous sommes appuyés sur les études réalisées sur des prairies temporaires en culture monospécifique ou associant un nombre restreint d'espèces comme «modèles» d'associations plus complexes pour mieux comprendre les mécanismes mis en jeu.

\section{2 / Diversité et valeur nutri- tive des prairies}

Pour les principales graminées et légumineuses fourragères sélectionnées et semées dans les prairies temporaires, les lois de variation de la digestibilité et donc de la valeur énergétique, de la valeur azotée et de l'ingestibilité sont relativement bien connues et ont fait l'objet de nombreuses publications notamment sous forme de tables (Andrieu et al 1981), remises à jour récemment (Baumont et al 2007). Les effets de la croissance et du stade phénologique ont été bien documentés. Pour les prairies permanentes et en particulier les prairies diversifiées, les études sont plus éparses du fait de la diversité des espèces végétales et de la diversité de composition botanique des couverts (Huyghe et Litrico 2008). Des travaux importants ont été cependant réalisés sur les prairies permanentes dans le massif alpin, en particulier dans les Alpes du Nord et en Suisse (Fleury et al 1990, Roumet et al 1996, Daccord et al 2006).

\section{1 / Description et compréhen- sion de la digestibilité des prai- ries permanentes}

La présence d'un grand nombre d'espèces dans une prairie permanente influence la digestibilité et la valeur nutritive de la biomasse produite, en raison des différences de digestibilité entre espèces à un même stade phénologique et des différences de stade de maturité entre espèces à une même date. Du fait de cette complexité et d'une moindre connaissance des espèces natives, les lois de réponse et la prévision de la valeur nutritive pour ces types de couverts végétaux ne sont pas encore clairement établies. La valeur nutritive des prairies diversifiées et leur valorisation par les animaux sont généralement plus faibles que celles de prairies conduites de façon intensive (Tallowin et Jefferson 1999, Bruinenberg et al 2002, Fiems et al 2004). Ces différences, en particulier pour la teneur en protéines et en minéraux, résultent cependant davantage d'une baisse ou d'une absence de fertilisation que de l'incidence de la diversité floristique sensu stricto. Au-delà de la valeur absolue de la valeur nutritive, plusieurs auteurs ont suggéré que la valeur nutritive de la prairie diversifiée était plus stable au cours du premier cycle (Gibon et al 1997, Bruinenberg et al 2002). Cette stabilité pourrait être expliquée i) par la contribution importante à la biomasse d'espèces à phénologie tardive qui conservent plus longtemps une proportion élevée de feuilles (partie la plus digestible d'une plante) et/ou de dicotylédones feuillues dont la valeur nutritive reste relativement stable au cours de la saison et ii) par le plus faible accroissement de biomasse au cours du temps et de l'existence d'une liaison négative entre la production de biomasse d'un couvert et la qualité de celle-ci (Huyghe et Litrico 2008). Duru (1997) a ainsi observé que les prairies de milieux pauvres, plus diversifiées, présentaient une digestibilité plus faible au stade précoce mais qui diminuait plus lentement au cours de la saison que celles de milieux riches, en raison de la présence d'espèces moins productives et à développement plus tardif. Daccord et al (2006) ont par ailleurs montré que la digestibilité ainsi que la valeur énergétique et azotée des prairies riches en dicotylédones feuillues étaient plus élevées et moins sujettes à diminution avec le stade de végétation que celles des prairies riches en graminées. Ces auteurs ont distingué quatre types principaux de prairies en fonction de leur proportion de graminées, de légumineuses et de diverses (figure 1). L'évolution de la

Figure 1. Evolution de la digestibilité (symboles en noirs reliés par des lignes pleines) et des teneurs en parois végétales (symboles en gris reliés par des tirets) pour 4 types de prairies définis par leur composition botanique, en fonction des stades de développement. Les stades de développement sont définis par les stades phénologiques atteints par quelques espèces de référence comme le dactyle et le pissenlit (d'après Daccord et al 2006).

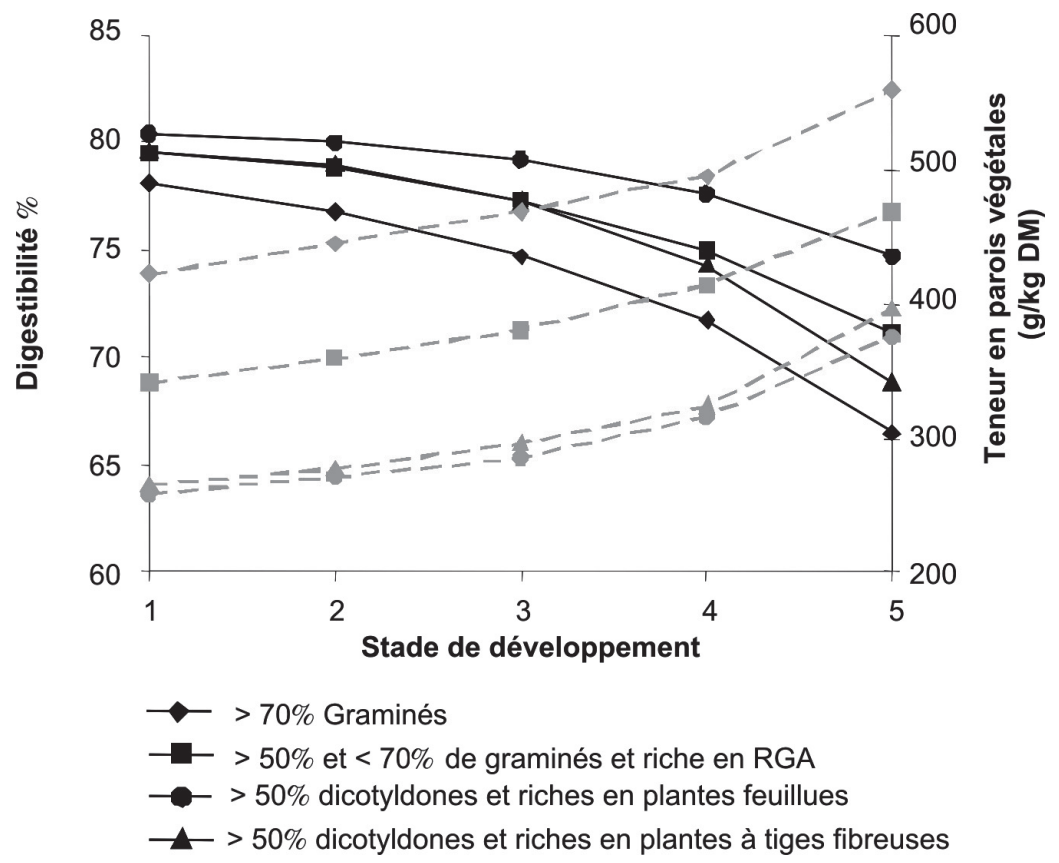


Figure 2. Relations entre la digestibilité mesurée avec la méthode pepsine-cellulase et $(A)$ la somme de temperature à la floraison (d'après Pontes et al 2007a), (B) le taux de matière sèche des feuilles pour 13 espèces de graminées natives des prairies permanents : Alopecurus pratensis (Ap), Anthoxanthum odoratum (Ao), Arrhenatherum elatius (Ae), Dactylis glomerata (Dg), Elytrigia repens (Er), Festuca arundinacea (Fa), Festuca rubra (Fr), Holcus lanatus (HI), Lolium perenne (Lp), Lolium perenne cultivar (Lpcv), Phleum pratense (Php), Poa pratensis (Pp), Poa trivialis (Pt) and Trisetum flavescens (Tf) (d'après Pontes et al 2007b).
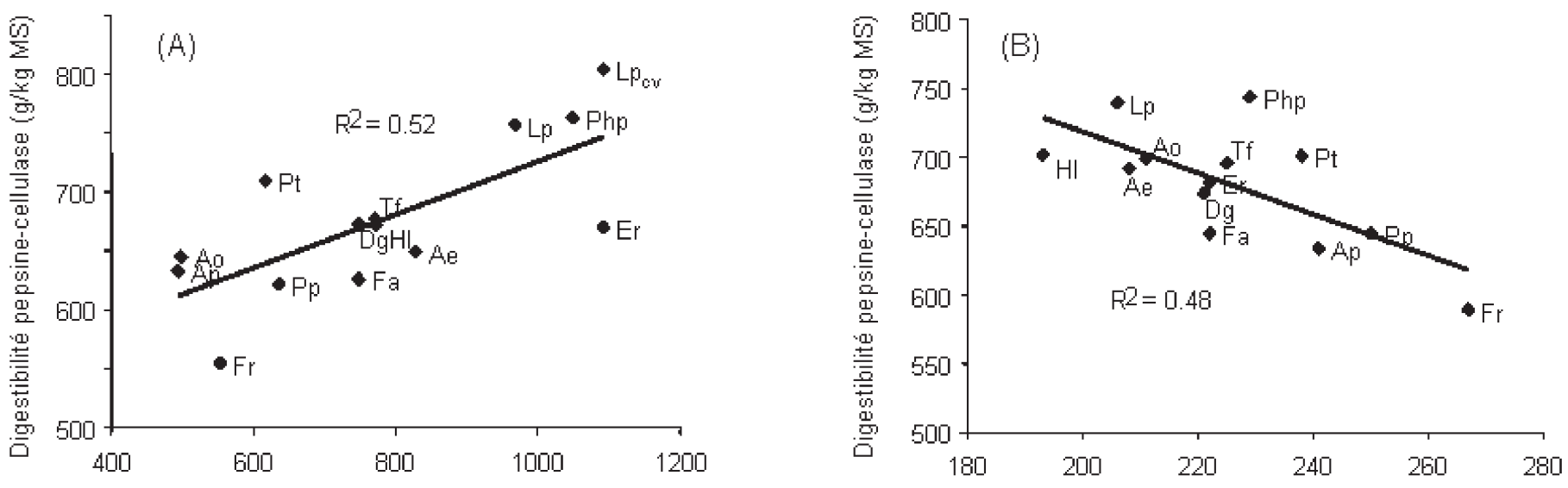

Somme de température à la floraison (Degrés jours depuis le 1er Mars, ${ }^{\circ} \mathrm{C}$ )

Teneur en matière sèche des limbes réhydratés (mgig)

composition chimique et de la valeur nutritive en fonction du stade de développement ont ensuite été calculées à partir de la valeur individuelle de chaque espèce pour des mélanges virtuels représentatifs des quatre types définis. Sur cette base, le groupe contenant plus de $50 \%$ de dicotylédones feuillues présente la digestibilité la plus élevée pour les 5 stades de développement suivi par le groupe riche en graminées avec le ray-grass anglais dominant. Les résultats obtenus récemment sur 180 prairies permanentes du Massif Central sont cohérents avec cette typologie (Rodrigues et al 2007). Dans cette étude, les prairies présentant une digestibilité élevée étaient caractérisées par une forte présence de dicotylédones feuillues comme le pissenlit et le céraiste (contribution à la biomasse aérienne : 15 et $3 \%$ respectivement), alors que les prairies de plus faible digestibilité étaient caractérisées par la présence de dicotylédones à tige fíbreuse comme la bistorte (contribution à la biomasse aérienne : $33 \%$ ).

Pour aller au delà de ces résultats, des travaux récents ont été engagés visant d'une part, une meilleure connaissance de la valeur et de la phénologie des espèces natives et d'autre part, intégrant l'approche fonctionnelle des espèces. Ainsi, la valeur nutritive de 13 graminées trouvées communément dans les prairies permanentes a été étudiée dans un essai factoriel avec 2 niveaux de fertilisation azotée et 2 fréquences de fauche (Pontes et al 2007a). La digestibilité cellulase variait en premier lieu avec l'espèce végétale, alors que la teneur en azote dépendait d'abord de la conduite (fertilisation et fréquence de fauche). Les différences de digestibilité entre espèces ont été en partie liées aux différences de phénologie, les espèces à phénologie tardive présentant des valeurs de digestibilité plus élevées au cours de la saison, confirmant ainsi l'importance de la prise en compte de ce facteur pour le premier cycle (figure 2A). S'il est en effet bien établi que la digestibilité des graminées diminue au cours du premier cycle de végétation avec l'avancée du stade de maturité (Andrieu et al 1981), cette diminution est d'autant plus rapide que l'espèce est précoce. Ainsi, lorsque l'on compare à même date la digestibilité du vulpin et celle du dactyle, elles sont équivalentes tant que les deux espèces sont au stade végétatif, mais lorsqu'on les compare 4 semaines après l'épiaison du dactyle, la digestibilité du vulpin, graminée très précoce, est alors de 10 points inférieure à celle du dactyle (Schubiger et al 2001). Les différences de valeur nutritive observées entre graminées peuvent par ailleurs s'interpréter à la lumière des groupes et traits fonctionnels. Les espèces orientées vers la capture des ressources, caractérisées par une croissance rapide, une surface d'échange avec le milieu élevée et un recyclage rapide des organes, développent moins de parois végétales et plus d'éléments cytoplasmiques comme les protéines. A même biomasse, leur teneur en parois végétales sera plus faible (Ansquer et al 2004) et la digestibilité de ces parois plus élevée (Al Haj Khaled et al 2006) que celle des espèces à stratégie de conservation des ressources. Avant la floraison, leur valeur alimentaire sera généralement plus élevée que celle des espèces à stratégie de conservation de la ressource, l'évolution du rapport feuilles/tiges pouvant réduire fortement ces différences par la suite. Les travaux de Rodrigues et al (2007) sur les prairies du Massif Central confirment que les écarts de digestibilité peuvent s'expliquer en partie par la contribution plus ou moins importante à la biomasse des graminées appartenant aux types fonctionnels de graminées compétitrices ou conservatives. En revanche, sur un réseau de parcelles d'éleveurs exploitées en fauche ou en pâture, Ansquer (2006) ne trouve pas de différence de digestibilité de la biomasse totale au pic de biomasse en fonction de la composition en types fonctionnels de graminées. D'après cet auteur, les graminées compétitives, à feuilles les plus digestibles (espèces de type compétitrices) sont aussi celles qui présentent le plus faible rapport feuilles/(tiges et gaines). A l'inverse, les espèces conservatives aux feuilles riches en matière sèche sont celles qui ont le rapport feuilles/(gaine et tiges) le plus élevé. Les deux paramètres observés évoluant globalement en sens inverse, les variations de digestibilité à l'échelle de la biomasse totale des différents types de prairies sont tamponnées. Enfin, les travaux de Louault et al (2005) à l'échelle de la communauté et de Pontès et al (2007b) pour les espèces confirment les liaisons entre la digestibilité et les traits foliaires associés à ces stratégies, illustrées sur la figure $2 \mathrm{~B}$ par la teneur en matière sèche des feuilles (encadré 2).

Concernant les dicotylédones, leur digestibilité est également très variable selon les espèces. La baisse de digestibilité des légumineuses au cours de la saison est plus faible que celle des gra- 


\section{Encadré 2. Protocole de mesure de la teneur en matière sèche des feuilles}

Des talles sont prélevées à l'aide d'un scalpel au niveau du sol sur la parcelle puis conservées dans un endroit froid. Les dernières feuilles adultes (entières et indemnes de toutes agression) de chaque talle sont ensuite coupées au laboratoire dans de l'eau de-ionisée puis placées à $4^{\circ} \mathrm{C}$ dans le noir pendant au moins 6 heures pour une complète réhydratation (Garnier et al 2001). Après réhydratation, les feuilles sont pesées en vert, séchées puis pesées en $\mathrm{sec}$. La matière sèche est ensuite calculée à partir du ratio poids sec sur poids vert.

minées, en particulier pour le trèfle blanc. La digestibilité de certaines espèces de diverses comme par exemple la renoncule rampante, peut être élevée et équivalente à celle du raygrass, qui présente la plus forte digestibilité dans la plupart des études (Korevaar 1986 cité par Bruinenberg et al 2002). De même, le pissenlit qui n'a que des feuilles quelle que soit la date, présente une digestibilité similaire à celle des graminées au stade végétatif, qui ne diminue pas avec l'avancée du stade de végétation (Schubiger et al 2001). A contrario, certaines dicotylédones de la famille des ombellifères caractérisées par des grosses tiges fibreuses, ainsi que d'autres espèces spécifiques de montagne de zones humides comme la renouée bistorte présentent des diminutions rapides de digestibilité avec l'avancée du stade de végétation (Daccord et al 2006). Pour les diverses, la classification fonctionnelle se base actuellement sur la présence ou non d'une tige au stade végétatif (Ansquer et al 2004). On distingue ainsi les dicotylédones qui, au stade végétatif, ne présentent pas de tiges mais seulement des feuilles larges (ou peu) découpées (par ex. pissenlit ou plantain), et les dicotylédones présentant des tiges (par ex. véronique), d'importants pétioles (par ex. anthrisque) ou des feuilles très découpées (par ex. achillée). Peu de travaux expérimentaux ont été encore engagés pour valider cette classification par rapport à la digestibilité mais les résultats obtenus par Daccord et al (2006) et Rodrigues et al (2007) ont confirmé son intérêt.

\section{2 / Valeur azotée}

La présence de certaines plantes peut également avoir un impact important sur la valeur azotée des fourrages. L'analyse d'une large base de données de mesures de dégradabilité dans le rumen a montré que la dégradabilité des protéines était moins élevée pour les fourrages issus de prairies permanentes que pour des fourrages monospécifiques de graminées ou de légumineuses (Nozières et al 2006). Ces résultats pourraient être dus à l'action de certains composés comme les tan- nins condensés, présents dans un certain nombre de légumineuses. En se complexant aux protéines, les tannins diminuent la dégradation et la solubilité de ces dernières, permettant ainsi un flux d'azote non ammoniacal plus important au niveau de l'intestin grêle et une meilleure efficacité de l'azote alimentaire (Min et al 2000). A titre d'exemple, dans des mélanges composés de luzerne et de sainfoin (respectivement pauvre et riche en tannins), la présence du sainfoin a réduit la solubilité des protéines de la luzerne (Julier et al 2002, Aufrère et al 2005). Par ailleurs, Selje et al (2007) ont testé l'aptitude de 500 plantes, prairiales ou non (fractions de plantes et huiles essentielles) à inhiber la protéolyse ruminale. Cinq plantes ont été particulièrement prometteuses dont 4 riches en tannins. La cinquième, une dicotylédone relativement commune en prairies permanentes (scabieuse des champs) a présenté un mécanisme d'action différent qui reste à élucider. D'autres travaux ont montré que la polyphénol oxydase, abondante par exemple dans le trèfle violet, et dans une moindre mesure dans le dactyle (Lee et al 2006) réduisait également, mais de façon indirecte, la protéolyse ruminale (Merry et al 2006, Aufrère et al 2008).

L'hypothèse selon laquelle les prairies permanentes diversifiées présentent une valeur nutritive moins élevée mais plus stable au cours de la saison que les prairies peu diversifiées n'a été validée que partiellement. La valeur nutritive et sa stabilité dépendent en premier lieu de la nature des espèces et de leur contribution à la biomasse. Actuellement, c'est l'abondance d'espèces à phénologie tardive ou bien l'abondance de dicotylédones feuillues qui expliquent cette plus grande stabilité plutôt que la richesse floristique ellemême. Par ailleurs, il est maintenant bien établi que certaines plantes et en particulier les plantes riches en tannins ont un impact sur la valeur azotée des fourrages suite à des interactions digestives, mettant ainsi en avant le rôle de l'identité spécifique plutôt que celui du nombre d'espèces.

\section{3 / Diversité alimentaire et stimulation de l'ingestion des animaux}

Au-delà de la valeur nutritive, la diversité floristique des ressources fourragères présente un intérêt en terme d'ingestion. Sur les végétations hétérogènes, les herbivores choisissent un régime alimentaire diversifié (Duncan et al 2003, Agreil et al 2005) et il a été suggéré que la diversité alimentaire pouvait être un stimulus positif pour l'animal qui augmente sa motivation à ingérer (Meuret et Bruchou 1994, Champion et al 2004). Cette hypothèse a été récemment validée expérimentalement, d'abord sur des génisses alimentées à l'auge avec des fourrages secs (Ginane et al 2002), puis avec des fourrages secs et humides (Baumont et Pomiès 2004), enfin sur des ovins pâturant des couverts prairiaux hétérogènes (Côrtes et al 2006). Cette dernière expérimentation a utilisé des associations simples comme «modèles» d'associations plus complexes et a été menée sur des animaux à faibles besoins (brebis taries). Il a ainsi été proposé à des brebis soit une prairie constituée d'une seule espèce végétale (ray-grass ou fétuque élevée), soit une prairie dans laquelle les deux espèces étaient associées en mélange «large» (deux demi-parcelles de chaque espèce) ou en mélange fin (imbrication des deux espèces), soit une prairie de raygrass et une prairie de fétuque successivement dans la journée. L'ingestion, aussi bien en termes de quantité totale qu'en termes d'éléments digestibles, ainsi que le temps de pâturage, ont été de 12 à 14\% plus élevés sur les traitements associant les deux espèces (quel que soit le type d'association) que sur la prairie de ray-grass (tableau 1). L'offre successive au cours de la journée de deux parcelles monospécifiques, mais constituées d'espèces végétales différentes a eu le même effet que leur offre simultanée avec deux surfaces adjacentes. La diversité alimentaire pourrait ainsi s'organiser à différentes échelles de temps, et l'étude de la cinétique des choix alimentaires au cours de la journée permettrait d'étayer une organisation à cette échelle. Il reste à étudier dans quelle mesure ces résultats sont généralisables à des situations où le temps de pâturage est moins «extensible», comme pour les animaux en lactation ou lorsque les disponibilités en herbe sont réduites. Les animaux à besoins plus élevés qui doivent pâturer plus longtemps avec une vitesse 
Tableau 1. Composition de la prairie et de la ration, et comportement alimentaire de brebis pâturant des monocultures de Ray-Grass $(R G)$, des monocultures de Fétuque Elevée $(F)$ ou des prairies associant les deux espèces végétales.

\begin{tabular}{|c|c|c|c|c|c|c|}
\hline Traitement & RGA & Fétuque & $\begin{array}{c}\text { Mélange } \\
\text { Large }\end{array}$ & Succession & $\begin{array}{l}\text { Mélange } \\
\text { fin }\end{array}$ & se \\
\hline $\begin{array}{l}\text { Proportion de ray-grass dans } \\
\text { la biomasse totale }(\%)\end{array}$ & 100 & 0 & 49 & 54 & 64 & \\
\hline $\begin{array}{l}\text { Proportion de ray-grass } \\
\text { dans la ration }(\%)\end{array}$ & 100 & 0 & $78 a$ & $78 a$ & $77 a$ & 7,1 \\
\hline Ingestion (g MO/j) & $1822 b$ & $1557 \mathrm{c}$ & $2029 a b$ & 2052 a & $2129 a$ & 359 \\
\hline $\begin{array}{l}\text { Temps de pâturage }(\mathrm{min} / \mathrm{j}) \\
\text { Vitesse d'ingestion }(\mathrm{g} \mathrm{MO} / \mathrm{min})\end{array}$ & $\begin{array}{r}366 \mathrm{c} \\
5.890 \mathrm{a}\end{array}$ & $\begin{array}{l}383 \mathrm{bc} \\
3.996 \mathrm{~b}\end{array}$ & $\begin{array}{r}420 \mathrm{ab} \\
4.900 \mathrm{a}\end{array}$ & $\begin{array}{l}397 \mathrm{abc} \\
5.004 \mathrm{a}\end{array}$ & $\begin{array}{r}430 \mathrm{a} \\
4.874 \mathrm{a}\end{array}$ & $\begin{array}{c}53 \\
0.901 \\
\end{array}$ \\
\hline
\end{tabular}

Traitement Mélange Large : les deux espèces végétales (RGA et Fétuque) sont offertes aux animaux sous forme de deux demi-parcelles, Traitement Succession : les deux espèces végétales sont offertes successivement aux animaux, la Fétuque de 9h à 16h et le Ray-Grass de 16h à 9h le lendemain matin,

Traitement Mélange Fin : les deux espèces sont finement imbriquées.

L'herbe était maintenue à $9 \mathrm{~cm}$, hauteur non limitante pour l'ingestion de l'animal.

Dans une même ligne, des valeurs suivies de lettres différentes sont significativement différentes $(P<0,05)(d$ 'après Côrtes et al 2006$)$.

d'ingestion plus élevée, ont en effet probablement moins d'élasticité dans leurs adaptations comportementales que les animaux à besoins peu élevés (Prache et al 1998). A titre d'illustration, la «relance», pratiquée par les bergers et qui consiste à conduire le troupeau sur une nouvelle zone de végétation (différente de celle sur laquelle les animaux sont en train de s'arrêter de pâturer), pour relancer leur ingestion, constitue une mise en pratique concrète de ce mécanisme de stimulation (Meuret 1993).

Ces expérimentations ont été réalisées avec seulement deux espèces. L'effet de la complexité et de la nature de la diversité floristique sur l'ingestion a été récemment étudié expérimentalement, mais avec des résultats variables. Des vaches alimentées avec des fourrages issus de prairies permanentes ont montré une ingestion supérieure lorsque la prairie était diversifiée par rapport à une prairie peu diversifiée (Bruinenberg et al 2003). De même, l'ingestion volontaire de moutons alimentés à l'auge avec de l'herbe coupée a été plus élevée lorsque l'herbe provenait d'une prairie diversifiée riche en dicotylédones plutôt que d'une prairie également diversifiée, mais surtout riche en graminées (contribution des dicotylédones à la biomasse : $16 v s$ 5\%; Andueza et al non publié). En revanche, Soder et al (2006) n'ont pas observé de différences de niveaux d'ingestion et de production laitière chez des vaches pâturant des associations végétales de niveau de complexité croissant (mélanges de 3, 6 ou 9 espèces végétales).

Ainsi, plusieurs études menées aussi bien chez des éleveurs qu'à partir d'expérimentations à l'auge et au pâturage ont démontré que, lorsque les herbivo- res domestiques ont le choix, ils choisissent un régime alimentaire diversifié. Cette diversité peut constituer un stimulus qui accroît la motivation de l'animal à ingérer et conduire à une augmentation de l'ingestion d'herbe via une augmentation du temps de pâturage.

\section{4 / Diversité floristique et caractéristiques sensorielles des produits laitiers et car- nés}

En dehors de l'effet bien connu de certaines plantes (crucifères, ail, oignon) sur la flaveur du lait ou du fromage (Urbach 1990), l'effet propre de la composition botanique et de la diversité floristique des fourrages sur les caractéristiques sensorielles (encadré 3) des fromages n'a été abordé que récemment. Les travaux réalisés depuis une dizaine d'années, dans le contexte des fromages au lait cru bénéficiant d'une Appellation d'Origine Contrôlée (AOC) en France et en Italie principalement, ont fait l'objet d'articles de synthèse récents (Coulon et al 2005, Martin et al 2005). Les données bibliographiques concernant spécifiquement l'effet de la composition botanique des prairies sur les caractéristiques sensorielles des produits carnés sont en revanche, rares et plus éparses. Les effets spécifiques de ce facteur sont plus difficiles à étudier dans la mesure où, contrairement au lait qui est produit en continu, les caractéristiques de la viande correspondent à des phénomènes d'accrétion qui ont lieu tout au long de la vie de l'animal. La plupart des études dans le domaine des produits carnés ont été réalisées en conditions expérimentales et se sont centrées sur l'effet d'une ou d'un nombre restreint d'espèces végétales plutôt que sur l'effet de la composition et de la diversité floristique.

\section{1 / Produits laitiers}

Les études, réalisées le plus souvent chez des producteurs de fromages fermiers (généralement à pâte pressée cuite ou demi-cuite), ont clairement fait apparaître des modifications qualitatives parfois très importantes des caractéristiques sensorielles des fromages concomitantes de changements de parcelles ou associées à des parcelles dont la composition botanique des prairies variait fortement : versant nord $v s$ versant sud, plaine vs montagne ou encore prairie monospécifique $v s$ prairie permanente (Coulon et Priolo 2002). La compilation de ces données (tableau 2) ne fait pas apparaître toutefois de tendances générales claires concernant les relations entre la diversité spécifique appréciée uniquement par le nombre d'espèces végétales et les caractéristiques sensorielles des différents types de fromages. Tout au plus peut-on observer que les fromages issus des par-

\section{Encadré 3. Appréciation des caractéristiques sensorielles des produits animaux}

Les caractéristiques sensorielles des fromages, des laits et des viandes sont appréciées à partir de l'évaluation de leur texture, de leurs odeurs (perçues directement par le nez), de leurs arômes (perçus lorsque le produit est en bouche grâce à la voie rétronasale), de leurs saveurs (perçues sur la langue) et de leur couleur, par un jury de dégustateurs, entraînés ou non. La flaveur est l'ensemble des sensations (texture, odeur, arôme et saveur) ressenties lors de la dégustation. 
Tableau 2. Différences $(p<0,05)$ de caractéristiques sensorielles de fromages issus d'animaux nourris avec des fourrages de composition floristique différente, à partir d'une compilation bibliographique. Italique : tendance $(p<0,10)$, NS : non significatif (F1: faciès 1 zone de pente, F2 : faciès 2 replat humide).

\begin{tabular}{|c|c|c|c|c|c|c|c|c|c|}
\hline \multirow[b]{2}{*}{ Auteurs } & \multirow[b]{2}{*}{ Fromage } & \multirow[b]{2}{*}{$\begin{array}{c}\text { Conserv. } \\
\text { Herbe }\end{array}$} & & \multirow[b]{2}{*}{ Diversité + } & \multirow[b]{2}{*}{ Diversité - } & \multicolumn{4}{|c|}{$\begin{array}{c}\text { Les fromages issus des parcelles les plus diversifiées } \\
\text { sont caractérisés par : }\end{array}$} \\
\hline & & & & & & Texture & Saveur & Odeurs & Arômes \\
\hline $\begin{array}{l}\text { Buchin } \\
\text { et al } 1999\end{array}$ & $\begin{array}{l}1 \text { producteur fermier } \\
\text { d'Abondance - } \\
2 \text { parcelles }\end{array}$ & Pâture & $\begin{array}{l}\text { Altitude } \\
\text { Nb Espèces } \\
\% \text { dicot. } \\
\% \text { gram. }\end{array}$ & $\begin{array}{l}1650 \text { (alpage) } \\
64 \\
57 \\
43 \\
\end{array}$ & \begin{tabular}{|l}
1650 (alpage) \\
$41(\mathrm{~F} 1)-25(\mathrm{~F} 2)$ \\
$47(\mathrm{~F} 1)-59(\mathrm{~F} 2)$ \\
$53(\mathrm{~F} 1)-16(\mathrm{~F} 2)$ \\
\end{tabular} & $\begin{array}{l}\text { + ferme, } \\
\text { + granuleuse, } \\
\text { - adhésive, } \\
\text { - souple, - fine }\end{array}$ & $\begin{array}{l}\text { - salé, } \\
\text { - amer, } \\
\text { - persistant }\end{array}$ & NS & $\begin{array}{l}\text { - aigre, + acide, } \\
\text { - fermenté, } \\
\text { + fruité, } \\
\text { - étable }\end{array}$ \\
\hline $\begin{array}{l}\text { Bugaud } \\
\text { et a/ } 2002\end{array}$ & $\begin{array}{l}3 \text { producteurs fermier } \\
\text { d'Abondance - } \\
10 \text { parcelles }\end{array}$ & Pâture & $\begin{array}{l}\text { Altitude } \\
\text { Nb Espèces } \\
\% \text { dicot. } \\
\% \text { gram. }\end{array}$ & $\begin{array}{l}1550-1800 \text { (alpage) } \\
52(47-60) \\
59(41-66) \\
35(27-42)\end{array}$ & $\begin{array}{l}950-1050 \text { (vallée) } \\
27(12-53) \\
31(14-53) \\
47(26-68)\end{array}$ & $\begin{array}{l}\text { + ferme, } \\
\text { - sableuse, } \\
\text { - élastique }\end{array}$ & - piquant & NS & $\begin{array}{l}\text { + fruité, } \\
\text { + animal }\end{array}$ \\
\hline $\begin{array}{l}\text { Martin } \\
\text { et al } 2005\end{array}$ & $\begin{array}{l}1 \text { producteur fermier } \\
\text { de Beaufort - } \\
2 \text { parcelles }\end{array}$ & Pâture & $\begin{array}{l}\text { Altitude } \\
\text { Nb Espèces } \\
\% \text { dicot. } \\
\text { \% gram. }\end{array}$ & $\begin{array}{l}2200 \text { (alpage) } \\
48 \\
62 \\
38 \\
\end{array}$ & \begin{tabular}{|l|}
2050 (alpage) \\
28 \\
53 \\
47 \\
\end{tabular} & NS & $\begin{array}{l}\text { - salée, } \\
\text { - acide }\end{array}$ & $\begin{array}{l}\text { - intense, } \\
\text { - animale }\end{array}$ & $\begin{array}{l}\text { - intense, } \\
\text { - diversifié, } \\
\text { - animal, } \\
\text { - épicé }\end{array}$ \\
\hline $\begin{array}{l}\text { Martin } \\
\text { et al non } \\
\text { publié }\end{array}$ & $\begin{array}{l}1 \text { producteur fermier } \\
\text { d'Abondance - } \\
2 \text { parcelles }\end{array}$ & Pâture & $\begin{array}{l}\text { Altitude } \\
\text { Nb Espèces } \\
\% \text { dicot. } \\
\text { \% gram. } \\
\end{array}$ & \begin{tabular}{|l|}
1020 (vallée) \\
53 \\
74 ( $21 \%$ ombellif.) \\
26 \\
\end{tabular} & \begin{tabular}{|l}
1060 (vallée) \\
34 \\
69 \\
31 \\
\end{tabular} & $\begin{array}{l}\text { + jaune, } \\
\text { + fondante, } \\
\text { - sableuse }\end{array}$ & $\begin{array}{l}\text { - salé, } \\
\text { - piquant, } \\
\text { + amer }\end{array}$ & $\begin{array}{l}\text { - brûlé, } \\
\text { - from. Bleu, } \\
\text { + pieds, } \\
\text { + terre }\end{array}$ & $\begin{array}{l}\text { - fruité, + alcool, } \\
\text { + herbacé, - soufre, } \\
\text { - vinaigre, } \\
\text { - apprécié }\end{array}$ \\
\hline $\begin{array}{l}\text { Verdier-Metz } \\
\text { et al } 2002\end{array}$ & $\begin{array}{l}\text { Expérim. } \\
\text { Saint-Nectaire }\end{array}$ & Foin & $\begin{array}{l}\text { Altitude } \\
\% \text { gram. }\end{array}$ & \begin{tabular}{|l|}
1100 \\
$70 \%$ (foin PP) \\
\end{tabular} & \begin{tabular}{|l|}
1100 \\
100 (Dactyle) \\
\end{tabular} & $\begin{array}{l}\text { - jaune, } \\
\text { - fondant }\end{array}$ & $\begin{array}{l}\text { - salé, } \\
\text { - amer }\end{array}$ & \begin{tabular}{|l|} 
- rance, \\
- moisi \\
\end{tabular} & - intense \\
\hline $\begin{array}{l}\text { Verdier-Metz } \\
\text { et al } 2000\end{array}$ & $\begin{array}{l}\text { Expérim. } \\
\text { Saint-Nectaire }\end{array}$ & Foin & $\begin{array}{l}\text { Altitude } \\
\text { Nb Espèces } \\
\text { \% gram. }\end{array}$ & $\begin{array}{l}1100 \\
27 \text { (foin PP) } \\
>65 \%\end{array}$ & $\begin{array}{l}1100 \\
1 \text { (Dactyle) } \\
100 \\
\end{array}$ & NS & NS & $\begin{array}{l}\text { + chou, } \\
\text { - cave }\end{array}$ & NS \\
\hline
\end{tabular}

celles les plus riches en espèces présentent des caractéristiques aromatiques légèrement moins fortes que ceux issus des parcelles les moins diversifiées lorsqu'ils sont dégustés au même âge. Selon les fromagers, ces types de fromages sont reconnus pour s'affiner plus lentement et une des hypothèses pour expliquer ce résultat pourrait être qu'ils n'ont pas encore pu développer tout leur potentiel aromatique. Une méta analyse réalisée sur une base de données composée de 50 fromages d'Abondance issus de 4 expérimentations différentes, dans lesquelles la composition botanique des prairies et les caractéristiques sensorielles des fromages pouvaient être mises en parallèle, a également été décevante quant à la mise en évidence d'associations entre les caractéristiques sensorielles des fromages, la diversité floristique et la présence de certaines plantes ou familles de plantes dans les prairies utilisées par les animaux (Martin et Farruggia, données non publiées). Ce résultat pourrait être dû au fort effet «expérimentation» englobant des effets année, stade de l'herbe (généralement mal caractérisé dans les études), conditions et durée d'affinage, etc., qui pourraient masquer les effets de la végétation.

Ainsi, avant même d'aborder l'effet spécifique de la diversité floristique, les effets de la composition botanique sur les propriétés sensorielles des fromages restent dans l'ensemble mal compris. L'ensemble des travaux ayant été réalisés sur des fromages au lait cru, il n'est pas exclu que la composition microbiologique des laits, connue pour jouer un rôle important dans la formation des caractéristiques sensorielles des fromages au lait cru, soit impliquée pour expliquer les effets de la composition botanique des fourrages, mais cette hypothèse n'a jamais été testée. Les hypothèses explorées à ce jour et décrites dans Coulon et Priolo (2002), concernent la présence dans le lait de composés issus directement des fourrages ingérés, ou bien produits par l'animal suite à l'ingestion de plantes particulières. Les travaux récents se sont particulièrement intéressés aux terpènes des plantes, qui, lorsque leur concentration est suffisante, ont des propriétés aromatiques et antimicrobiennes reconnues. Toutefois, dans deux essais récents réalisés sur des fromages à pâte pressée (Tornambe et al 2007a) ou à pâte pressée cuite (Buchin et al données non publiées), l'ajout de terpènes exogènes dans le lait avant la fabrication n'a eu que des effets très marginaux sur le développement des différentes populations microbiennes ou sur la production de composés volatils dans les fromages. Les effets de la composition botanique des prairies sur les composés volatils et sur les caractéristiques sensorielles des fromages sont donc certainement indépendants des terpènes. D'autres hypothèses concernent la plasmine, une protéase endogène provenant du sang, qui joue un rôle important dans le processus d'affinage des fromages à pâte pressée cuite. L'augmentation de la concentration en plasmine observée avec certains régimes alimentaires pourrait être due à l'ingestion de certaines espèces végétales telles que les renoncules (Buchin et al 1999, Bugaud et al 2001). Dans ce cas, ce n'est donc pas la diversité floristique qui interviendrait sur cette composante sensorielle, mais la présence d'une espèce ou d'une famille d'espèces-clé faisant à nouveau référence au concept d'identité spécifique. Cette hypothèse reste cependant à confirmer. Les acides gras du lait sont aussi vraisemblablement impliqués dans l'effet de la composition botanique des prairies sur la texture des fromages, dans la mesure où les proportions d'acides gras longs et polyinsaturés qui ont un point de fusion plus bas, sont plus élevées dans les fromages de montagne que de plaine (Bugaud et al 2001, Collomb et al 2002a, Zeppa et al 2003).

Enfin, concernant les caractéristiques sensorielles du lait cru, les effets de la diversité floristique des prairies n'ont pas pu être mis en évidence dans deux études récentes : l'une menée chez des producteurs de lait (Guichard et al 2006), l'autre en conditions contrôlées (Tornambe et al 2007b). Cette absence d'effet pourrait avoir plusieurs origines : $i$ ) le lait est plus difficile à déguster que les fromages, $i$ ) la fabrication fromagère concentre la majorité des composants du lait, matières grasses et composés liposolubles notamment et iii) les mécanismes évoqués précédemment pour le fromage impliquent des composés du lait intervenant au cours de la fabrication et de l'affinage, phase absente dans le cas du lait.

En conclusion, les résultats expérimentaux ont mis en évidence des liens 
entre la composition botanique des prairies et les caractéristiques sensorielles des fromages, même si les mécanismes sous-jacents ne sont pas élucidés et semblent complexes. La compilation de ces résultats n'a cependant pas établi de tendances fortes entre la diversité floristique appréciée par le nombre total d'espèces et les caractéristiques sensorielles des fromages. Indépendamment du niveau de diversité floristique, la seule présence d'une espèce ou d'une famille d'espèces dans une prairie pourrait intervenir dans les processus d'élaboration des caractéristiques sensorielles des fromages. Par ailleurs, des études récentes ont montré que la diversité floristique n'avait pas d'effet sur les caractéristiques sensorielles des laits crus.

\section{2 / Produits carnés}

De nombreux travaux ont montré que la viande des bovins et des ovins finis à l'herbe est plus sombre, moins tendre et a une flaveur plus forte que la viande des animaux finis avec des régimes à base de concentrés (Coulon et Priolo 2002). S'intéressant plus spécifiquement à la nature de la prairie, certains auteurs ont comparé les caractéristiques sensorielles de la viande de bovins et d'ovins engraissés sur différentes prairies monospécifiques ou sur des associations composées d'un faible nombre d'espèces. Larick et al (1987) ont ainsi comparé la viande de bouvillons abattus après une période de pâturage de 7 mois sur 3 pâtures différentes, composées chacune de 1 ou 2 espèces différentes (fétuque élevée et brome inermis ; trèfle violet et dactyle ; trèfle violet). Les dégustateurs n'ont pas relevé de différences significatives. Pour la viande ovine, plusieurs travaux montrent que la viande d'agneaux élevés au pâturage présente une flaveur plus intense et moins appréciée lorsque l'animal consomme un régime riche en légumineuses par rapport à un régime riche en graminées. Deux légumineuses, en particulier, sont pointées : le trèfle blanc et la luzerne. Le trèfle blanc conduit à des concentrations en indole et en scatole dans le tissu adipeux plus élevées que le ray-grass anglais (Schreurs et al 2007a). Ces composés, qui sont responsables de flaveurs et d'odeurs désagréables, sont formés dans le rumen à partir de la déamination et de la décarboxylation microbienne du tryptophane, et leur occurrence est donc accrue lorsque les protéines du fourrage présentent une solubilité élevée et une dégradation rapide dans le rumen (Schreurs et al 2007a). Les tannins condensés de cer- taines légumineuses, telles que le lotier corniculé, le sulla et le lotier pédonculé, diminuent la biosynthèse ruminale de ces composés odorants, car ils ralentissent la dégradation ruminale des protéines (Schreurs et al 2007b). Ainsi, il a été observé que les concentrations en indole et scatole dans le liquide ruminal, dans le plasma et dans le tissu adipeux intermusculaire étaient plus faibles chez des agneaux qui pâturaient du lotier corniculé que chez des agneaux qui pâturaient des prairies de ray-grass anglais/trèfle blanc (Schreurs et al $2007 \mathrm{c}$ ). Pour ce qui concerne la luzerne, Park (1972) a observé que la viande d'agneaux pâturant de la luzerne était moins appréciée et présentait plus souvent des flaveurs atypiques que celle d'agneaux pâturant du phalaris (Phalaris tuberosa). Cet effet pourrait s'expliquer par une concentration plus importante d'acides gras courts (C8 et C9) ramifiés observés dans la viande d'agneaux nourris avec de la luzerne (Young et al 2003). Au-delà de ces deux légumineuses, Young et al (1994) ont comparé la flaveur de la viande d'agneaux âgés de 6 mois qui avaient pâturé pendant 6 semaines 7 parcelles différentes, composées d'une seule espèce : phalaris (Phalaris aquatica), luzerne, chicorée, «herbe de prairie» (Bromus wildenowii), ray-grass anglais, fétuque élevée ou dactyle. La viande d'agneau produite sur le phalaris a été plus tendre et a présenté une intensité de flaveurs atypiques, telles que «poisson» et «rance» plus élevée, vraisemblablement en raison des alkilamines d'indole de cette plante, qui pourraient causer des perturbations métaboliques aux animaux se répercutant sur la flaveur de la viande (Sheath et al 2001). Par ailleurs, une synthèse récente (Priolo et Vasta 2007) fait état de l'effet de l'ingestion de pulpe de caroube, de feuilles d'acacia, ou de sulla (plantes riches en tannins) sur la couleur plus claire des viandes. Les tannins seraient en effet responsables d'une diminution de la biosynthèse ruminale de la vitamine B12, un précurseur des pigments héminiques. Enfin, Whittington et al (2006) ont montré des différences de flaveur de viande d'agneaux ayant pâturé des types de couverts végétaux très différents. Ils ont comparé de la viande d'agneaux de race Suffolk Mule engraissés (pendant au moins 100 jours) sur une prairie peu diversifiée à base de ray-grass anglais (traitement témoin ; 10 espèces végétales) ou bien sur des prés-salés (31 espèces végétales) à de la viande d'agneaux de race Scottish Blackface engraissés sur des landes à bruyères (51 espèces végéta- les) ou sur des parcours très diversifiés (60 espèces végétales). Le gras des agneaux engraissés sur les parcours les plus diversifiés présentait plus d'odeurs anormales et la flaveur «agneau» y avait été jugée plus forte. L'interprétation des résultats de cet essai reste toutefois délicate du fait $i$ ) de possibles confusions d'effets liés notamment aux différences de race ii) du rôle éventuel de l'identité spécifique de quelques espèces présentes sur les parcours diversifiés plutôt que de la diversité du couvert lui-même.

Les études sont encore peu nombreuses dans ce domaine. Elles ont surtout pointé le rôle de l'identité spécifique en mettant en évidence l'apparition d'effets indésirables sur la flaveur de la viande due à la présence d'une espèce végétale, comme par exemple le trèfle blanc et la luzerne, ou l'influence des tannins sur la réduction de la biosynthèse ruminale de composés responsables de flaveurs désagréables ou de la couleur. Le rôle de la diversité floristique bien qu'exploré dans une étude n'a pu être explicitement démontré.

\section{5 / Diversité floristique et caractéristiques nutrition- nelles des produits laitiers et carnés}

Trois types de composés jouant un rôle reconnu ou supposé sur la valeur nutritionnelle des produits animaux pour la santé humaine sont abordés dans cette partie : les acides gras, les caroténoïdes et les polyphénols. Les acides gras font l'objet d'un nombre extrêmement important de publications scientifiques récentes, d'origine géographique très variée, portant sur leur concentration dans les rations et dans les produits animaux selon l'alimentation. La composition en acides gras du lait selon la nature des prairies a, en revanche, été étudiée essentiellement en France, en Suisse et en Belgique, avec une synthèse récente sur les produits laitiers (Chilliard et al 2007). Très peu de travaux ont été menés pour ce qui concerne l'effet de la diversité floristique sur les caractéristiques nutritionnelles de la viande, et à notre connaissance, il n'y a pas eu d'étude sur les bovins. Concernant les caroténoïdes et les polyphénols, les études en lien avec les prairies sont encore très rares. Leur intérêt nutritionnel est peu connu, et les recherches en relation avec la flore des prairies en sont à leurs balbutiements. 
Encadré 4. Rappel sur la provenance et les étapes de transformations des Acides Gras (AG) dans le rumen et la mamelle

Principales voies de synthèse des acides gras trans et du CLA du lait (d'après Chilliard et al 2000)

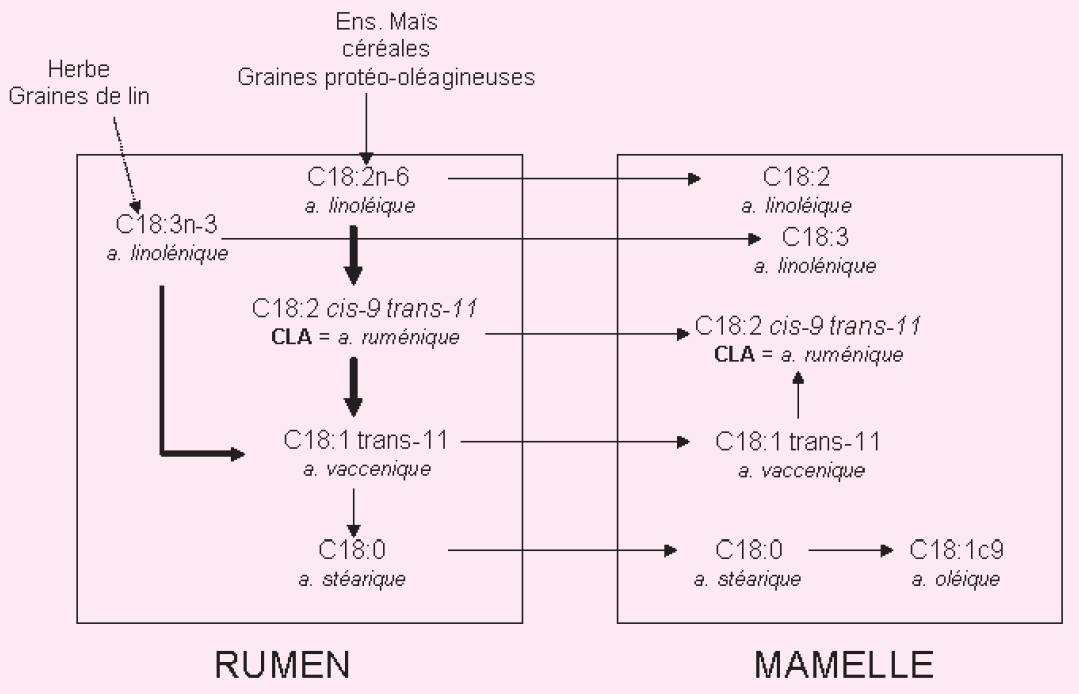

Les acides linoléique et linolénique sont isomérisés et biohydrogénés dans le rumen pour aboutir à la production de nombreux isomères du C18:2 et du C18:1, en particulier des CLA et des acides gras trans (dont l'acide vaccénique), et in fine au C18:0 (acide stéarique). Le CLA cis-9 trans -11 (acide ruménique) représente 80 à $90 \%$ des CLA des laits des ruminants (Bauman et al 2001). La biohydrogénation est dépendante de l'environnement ruminal $(\mathrm{pH})$ et peut être fortement affectée par la composition de la ration (quantité de concentré, teneur en amidon, nature des fourrages...). Dans des conditions normales, elle est intense puisque le pourcentage de disparition des acides gras 18:2 n-6 et 18:3 n-3 entre la bouche et le duodénum des ruminants est en moyenne respectivement de 80 et $92 \%$ (Glasser et al 2008). Tous ces AG sont ensuite absorbés au niveau de l'intestin, prélevés au niveau de la mamelle où ils sont soit sécrétés directement dans le lait, soit désaturés en particulier par l'activité la delta-9-désaturase qui ajoute une double liaison cis-9 à différents AG et qui renverse ainsi l'effet de la biohydrogénation ruminale (Griinari et al 2000). Environ 90\% de l'acide ruménique du lait provient de la désaturation mammaire de l'acide vaccénique.

\section{1 / Les acides gras}

Les produits laitiers et carnés des ruminants sont généralement riches en acides gras saturés, et dans le cas des fromages, riches en sel. Pour cette raison, ils sont considérés, lorsqu'ils sont consommés en excès, comme des facteurs de risque pour les maladies cardiovasculaires (Grant 1998). Bien que pauvres en acides gras polyinsaturés, ils contiennent néanmoins des acides gras insaturés de la série n-3 dits «oméga 3» et des acides gras spécifiques comme les acides linoléiques conjugués (CLA ou acide ruménique) dont les effets protecteurs vis-à-vis des maladies cardiovasculaires ont été avancés. L'intérêt nutritionnel de certains acides gras spécifiques des produits laitiers et carnés n'a cependant été mis en évidence que sur des modèles animaux. Des effets hypocholestérolémiants des acides vaccénique et ruménique du lait ont été observés chez le lapin (Bauchart et al 2007). Des résultats positifs sur des modèles cellulaires commencent également à apparaître comme par exemple la mise en évidence d'effets antitumoraux des CLA de la viande (De la Torre et al 2005, 2006). Par ailleurs, les acides gras insaturés dits «trans» des produits issus des ruminants seraient plutôt bénéfiques pour la santé humaine, contrairement à ceux de nombreuses margarines (Chardigny 2007). Ces constatations incitent à orienter les conduites d'élevage de manière à optimiser la valeur nutritionnelle des produits laitiers et carnés des ruminants. La composition en acides gras peut être en effet modulée par l'alimentation de l'animal (Chilliard et al 2008). En particulier, il est possible d'accroître la teneur en «oméga 3», en CLA et en acides gras trans, la concentration de ces deux familles d'acides gras étant positivement corrélée (Chilliard et al 2007). L'accent est mis, dans cette partie, sur les variations de la teneur de ces acides gras successivement, dans les fourrages, dans les produits laitiers puis dans les produits carnés, en fonction du type de prairies pâturées par les animaux. L'encadré 3 rappelle succinctement la provenance et les étapes de transformations des acides gras dans le rumen et la mamelle (encadré 4). Une compilation des données bibliographiques explicitant la nature des prairies et les teneurs en acides gras de l'herbe, des laits et des viandes correspondants est présentée dans les tableaux 4 et 5 .

\section{a) Dans les fourrages et le rumen}

Les fourrages à base d'herbe, ainsi que la graine de lin, sont une source majeure d'acide linolénique (C18:3 n-3 de la famille des oméga 3 ) pour les animaux. L'herbe verte contient de 1 à $3 \%$ (de la matière sèche : MS) d'acides gras totaux constitués de 50 à $75 \%$ d'acide linolénique. Les valeurs les plus élevées sont observées pour l'herbe de printemps et les repousses d'automne (Chilliard et al 2007). Une vache consommant $20 \mathrm{~kg}$ de MS d'herbe jeune ingère ainsi autant d'acide linolénique qu'une vache recevant un régime à base d'ensilage de maïs avec $1 \mathrm{~kg}$ de graine de lin. Pour cette raison, les régimes riches en herbe permettent un accroissement significatif des teneurs en acide linolénique du lait (Chilliard et al 2001) et de la viande (Nuernberg et al 2005), par rapport à des rations classiques non complémentées en suppléments lipidiques riches en oméga 3. Une analyse des données de la bibliographie montre que le ray-grass anglais a une proportion d'acide linolénique plus forte que d'autres espèces, et plus forte que la prairie permanente (RGA : 66,2 vs prairies permanentes : $53,8 \%$ des acides gras totaux, tableau 3). Il peut $\mathrm{y}$ avoir des biais à ce résultat, en 
Tableau 3. Teneurs en acides gras totaux, en acide linoléique et en acide linolénique : médianes, 1er et 3ème quintiles, issues d'une compilation de la bibliographie (Glasser et Doreau données non publiées)

\begin{tabular}{|c|c|c|c|c|c|c|c|c|c|c|}
\hline \multirow[b]{2}{*}{ Fourrage } & \multirow[b]{2}{*}{$\begin{array}{c}\text { Nombre } \\
\text { de données }\end{array}$} & \multicolumn{3}{|c|}{ AG totaux (en \% MS) } & \multicolumn{3}{|c|}{$\begin{array}{c}\text { Acide linoléique } \\
\text { (\% des } A G)\end{array}$} & \multicolumn{3}{|c|}{$\begin{array}{c}\text { Acide linolénique } \\
(\% \text { des } A G)\end{array}$} \\
\hline & & Médiane & Q1 & Q3 & Médiane & Q1 & Q3 & Médiane & Q1 & Q3 \\
\hline Ray-grass & 109 & 2,31 & 1,76 & 2,87 & 11,8 & 10,1 & 13,4 & 66,2 & 59,9 & 72,1 \\
\hline Dactyle & 35 & 1,47 & 1,13 & 2,08 & 16 & 14,6 & 17,4 & 53,5 & 45,5 & 57,8 \\
\hline Fléole & 19 & 1,88 & 1,63 & 2,08 & 21,6 & 19,7 & 23,2 & 48 & 46,1 & 50,2 \\
\hline Trèfle blanc & 19 & 3,18 & 2,81 & 4,38 & 16,3 & 13,9 & 17 & 47,1 & 40 & 55,5 \\
\hline Trèfle violet & 11 & 2,07 & 1,4 & 2,19 & 21,4 & 19,5 & 23,1 & 43 & 42,5 & 45 \\
\hline Luzerne & 8 & 1,71 & 1,61 & 1,76 & 21,4 & 19,4 & 22,4 & 39,1 & 38,5 & 40,7 \\
\hline $\begin{array}{c}\text { Prairies } \\
\text { permanentes }\end{array}$ & 31 & 2,62 & 2,04 & 3,5 & 15,5 & 12,9 & 17,4 & 53,8 & 48,5 & 56,4 \\
\hline
\end{tabular}

raison de la diversité des conditions expérimentales, mais la tendance est confirmée par quelques comparaisons directes (Morel et al 2006, Lourenço et al 2007, Tornambé et al 2007b, tableaux 4 et 5). De même, Clapham et al (2005) ont observé, sur des fourrages ayant poussé dans les mêmes conditions sous serre, que la teneur en acide linolénique était plus élevée pour des graminées que pour des diverses. Enfin, une diminution de la part des graminées et des légumineuses au profit d'autres familles botaniques (ombellifères, géraniacées) entraîne une diminution de l'acide linolénique, et l'apparition d'acides gras mineurs rares, tels le C16:3 (Bugaud et al 2000). Les pâturages d'altitude ont pour leur part une teneur en acides gras totaux et en acide linolénique plus élevées que les fourrages de plaine (Collomb et al 1999). Il n'y a toutefois pas de relation systématique entre l'altitude des pâturages et la teneur en acides gras ou la proportion d'acide linolénique. L'effet observé tient probablement à la différence de maturité de l'herbe liée à l'altitude, à une même date.

Avant d'arriver dans la mamelle ou les muscles, les acides gras ingérés à partir des fourrages sont remaniés au niveau du rumen (encadré 4). Pour une même teneur initiale, ces acides gras peuvent être plus ou moins biohydrogénés dans le rumen. Des composés présents dans certaines espèces peuvent en effet diminuer la biohydrogénation et donc augmenter la concentration en acide linolénique non transformé dans le rumen (Doreau et al 2005, Lee et al 2006). C'est le cas de diverses variétés de trèfle violet par exemple, riches en polyphénol oxydase, une enzyme qui transforme certains acides phénoliques en quinones qui inhibent la protéolyse et la lipolyse bactérienne dans le rumen.

\section{b) Dans les produits laitiers}

Les teneurs en acides gras polyinsaturés présents dans l'herbe ne reflètent pas toujours celles observées dans le lait. Ainsi, à l'inverse des résultats observés sur les fourrages, des corrélations ont montré que la teneur en acide linolénique du lait de vache était plus faible lorsque les graminées étaient majoritaires, et plus élevée avec certai-

Tableau 4. Comparaison des teneurs en acides gras (en \% des AG totaux) de la prairie et des laits correspondants, en fonction de la composition floristique de la prairie (à partir d'une compilation bibliographique).

Gra (RGA) : graminées dont ray-grass anglais ; Leg : légumineuses ; Div : diverses ; Ns : nombre d'espèces recensées ; Is : indice de Shannon; MG : matière grasse ; AGS : somme des acides gras saturés; AGMI : somme des acides gras monoinsaturés; AGPI: somme des acides gras polyinsaturés ; C18:2 : acide linoléique ; C18:3 : acide linolénique ; C18:1t11 : acide vaccénique ; CLA : acide linoléique conjugué C18:2c9t11 ; * : somme des CLA ; ${ }^{* *}$ : C18:1t10 + C18:1t11; *** : tous isomères du C18:2.

\begin{tabular}{|c|c|c|c|c|c|c|c|c|c|c|c|c|c|c|}
\hline \multirow{2}{*}{ Auteur } & \multirow{2}{*}{ Intitulé des prairies } & \multicolumn{3}{|c|}{ Caractéristique de la prairie } & \multicolumn{3}{|c|}{ Herbe } & \multicolumn{7}{|c|}{ Lait } \\
\hline & & Gra (RGA)/Leg/Div & Ns & Is & $\% \mathrm{MG}$ & C18:2 & C18:3 & AGS & AGMI & AGPI & C18:2 & C18:3 & C18:1t11 & CLA \\
\hline \multirow{2}{*}{ Morel et al 2006} & Trèfle violet en culture pure & & - & - & - & 15,1 & 64,4 & 58 & 29 & 4,5 & 1,3 & 0,8 & 1,9 & 1,0 * \\
\hline & Prairie très riche en graminées & $99(36) / 1 / 0$ & - & - & - & 19,9 & 55,3 & 59 & 30 & 5,0 & 1,6 & 1,1 & 1,7 & $0,9^{*}$ \\
\hline \multirow{3}{*}{$\begin{array}{l}\text { Tornambé et al } \\
2007 \mathrm{~b}\end{array}$} & Prairie peu diversifiée & $76(29) / 7 / 17$ & 17 & 2,3 & 2,0 & 14,0 & 61,2 & - & - & - & $0,84 b$ & $0,63 a b$ & $2,81 \mathrm{~b}$ & 1,88 \\
\hline & Prairie moyennement diversifiée & $74(0) 19$ & 31 & 3,7 & 1,8 & 14,6 & 60,9 & - & - & - & $0,76 \mathrm{c}$ & $0,57 \mathrm{~b}$ & $3,10 \mathrm{ab}$ & 1,73 \\
\hline & Prairie très diversifiée & $52(0) / 2 / 46$ & 50 & 4,1 & 1,6 & 18,7 & 54,0 & - & - & - & $1,04 a$ & $0,72 a$ & $3,62 a$ & 1,62 \\
\hline \multirow{3}{*}{$\begin{array}{l}\text { Collomb et al } \\
2002 b\end{array}$} & Vallée & $65 / 36 / 0$ & 6 & 2,1 & - & - & - & - & - & - & $1,1 \mathrm{~b}$ & $0,8 \mathrm{~b}$ & $2,1 c^{\star *}$ & $0,8 \mathrm{c}$ \\
\hline & Montagne & $65 / 7 / 29$ & 52 & 4,1 & - & - & - & - & - & - & $1,4 a$ & $0,8 b$ & $3,7 \mathrm{~b}^{* *}$ & $1,5 b$ \\
\hline & Haute montagne & $42 / 10 / 48$ & 53 & 4,3 & - & - & - & - & - & - & $1,3 a$ & $1,2 \mathrm{a}$ & $5,1 a^{* *}$ & $2,2 \mathrm{a}$ \\
\hline \multirow{2}{*}{$\begin{array}{l}\text { Bugaud et al } \\
2000\end{array}$} & Vallée (3 prairies) min et max & $\begin{array}{l}29 / 19 / 15 \\
63 / 26 / 43\end{array}$ & $\begin{array}{l}16 \\
29\end{array}$ & - & $\begin{array}{l}2,4 \\
2,9\end{array}$ & $\begin{array}{l}17,9 \\
25,4\end{array}$ & $\begin{array}{l}42,8 \\
53,2\end{array}$ & - & - & - & $\begin{array}{l}4,2^{\star \star \star} \\
4,5^{\star \star *}\end{array}$ & $\begin{array}{l}0,8 \\
1,1\end{array}$ & - & - \\
\hline & $\begin{array}{l}\text { Montagne (4 prairies) } \\
\text { min et max }\end{array}$ & $\begin{array}{c}39 / 4 / 22 \\
54 / 24 / 58 \\
\end{array}$ & $\begin{array}{l}23 \\
26\end{array}$ & - & $\begin{array}{l}1,9 \\
2,2 \\
\end{array}$ & $15,118,1$ & $47,153,8$ & - & - & - & $\begin{array}{l}5,4^{\star \star \star} \\
7,3^{\star * *}\end{array}$ & $\begin{array}{l}1,3 \\
1,6\end{array}$ & - & - \\
\hline \multirow{2}{*}{$\begin{array}{l}\text { Lourenço et al } \\
2005\end{array}$} & Prairie peu diversifiée (Ens.) & $96(6) / 0 / 4$ & - & - & 1,2 & 13,9 & 39,1 & - & - & - & $0,86 a$ & $0,55 a$ & $0,50 a$ & $0,26 a$ \\
\hline & Prairie diversifiée (Ens.) & $55(4) / 11 / 34$ & - & - & 1,3 & 14,6 & 38,6 & - & - & - & $0,95 b$ & $0,59 \mathrm{~b}$ & $0,82 b$ & $0,44 b$ \\
\hline \multirow{3}{*}{$\begin{array}{l}\text { Guichard et al } \\
2006\end{array}$} & $\begin{array}{l}\text { Prairies peu diversifiées } \\
\text { à graminées }\end{array}$ & $88(10) / 6 / 6$ & - & - & - & - & - & 66,6 & 29,1 & $4,4 a$ & $1,3 a$ & 0,8 & 4,3 & $1,7 a$ \\
\hline & \begin{tabular}{|l|} 
Prairies diversifiées \\
à plantes aromatiques \\
\end{tabular} & $66(13) / 8 / 27$ & - & - & - & - & - & 66,8 & 28,8 & $4,4 a$ & $1,4 a$ & 0,9 & 4,2 & $1,5 a$ \\
\hline & $\begin{array}{l}\text { Prairies diversifiées } \\
\text { à légumineuses }\end{array}$ & $59(5) / 20 / 21$ & - & - & - & - & - & 64,3 & 30,4 & $5,3 b$ & $1,5 b$ & 0,9 & 5,4 & $2,2 \mathrm{~b}$ \\
\hline \multirow{2}{*}{$\begin{array}{l}\text { Astier et al } \\
\quad 2004\end{array}$} & $\begin{array}{l}\text { Prairies de ray-grass } \\
\text { de Bretagne }\end{array}$ & - & - & - & - & - & - & - & - & - & - & - & - & $0,73 a$ \\
\hline & $\begin{array}{l}\text { Prairies permanentes } \\
\text { d'Auvergne }\end{array}$ & - & - & - & - & - & - & - & - & - & - & - & - & $1,44 b$ \\
\hline
\end{tabular}


Tableau 5. Comparaison des teneurs en acides gras (en \% des AG totaux) de viandes issues d'animaux ayant pâturé des prairies de compositions floristiques variables (à partir d'une compilation bibliographique).

Gra (RGA) : graminées dont ray-grass anglais ; Leg : légumineuses ; Div : diverses ; Ns : nombre d'espèces recensées ; Is : indice de Shannon ; MG : matière grasse ; AGS : somme des acides gras saturés ; AGMI : somme des acides gras monoinsaturés ; AGPI : somme des acides gras polyinsaturés ; C18:2 : acide linoléique ; C18:3 : acide linolénique ; C18:1t11 : acide vaccénique ; CLA : acide linoléique conjugué ; * : $18: 1 \mathrm{t} 10+\mathrm{C} 18: 1 \mathrm{t} 11 ;{ }^{* *}$ : en \% des AGPI du muscle semi-membranosus.

\begin{tabular}{|c|c|c|c|c|c|c|c|c|c|c|c|c|c|c|}
\hline \multirow{2}{*}{ Auteur } & \multirow{2}{*}{$\begin{array}{l}\text { Intitulé des } \\
\text { prairies }\end{array}$} & \multicolumn{3}{|c|}{$\begin{array}{c}\text { Caractéristique de } \\
\text { la prairie }\end{array}$} & \multicolumn{3}{|c|}{ Herbe } & \multicolumn{7}{|c|}{ Viande } \\
\hline & & $\begin{array}{l}\text { Gra }(R G A) / \\
\text { Leg/Div }\end{array}$ & Ns & Is & $\stackrel{\%}{M G}$ & C18:2 & C18:3 & AGS & AGMI & AGPI & C18:2 & C18:3 & $\begin{array}{l}\text { C18: } \\
1 \mathrm{t} 11\end{array}$ & $\begin{array}{l}\text { CLA } \\
\text { C9t11 }\end{array}$ \\
\hline \multirow{2}{*}{$\begin{array}{c}\text { Adnoy et al } \\
2005\end{array}$} & Plaine & - & - & - & - & - & - & 45,3 & $40,3 a$ & $4,4 a$ & & & & \\
\hline & Montagne & - & - & - & - & - & - & 45,9 & $38,2 b$ & $6,9 b$ & & & & \\
\hline \multirow{3}{*}{$\begin{array}{c}\text { Louranço } \\
\text { et al } \\
2007 a\end{array}$} & $\begin{array}{c}\text { Prairie riche en } \\
\text { Ray-grass } \\
\text { anglais }\end{array}$ & $\begin{array}{c}91 \\
(69) / 0 / 0\end{array}$ & - & - & 3,7 & $13,9 b$ & $57,2 a$ & 53,4 & 35,9 & $4,55 b$ & $0,86 c$ & $1,50 \mathrm{~b}$ & $4,47 a^{*}$ & $1,01 b$ \\
\hline & $\begin{array}{l}\text { Prairie riche en } \\
\text { légumineuses }\end{array}$ & $\begin{array}{c}33 \\
(19) / 61 / 0 \\
\end{array}$ & - & - & 3,7 & $17,3 a$ & $52,0 \mathrm{~b}$ & 50,3 & 35,3 & $7,78 a$ & $2,35 a$ & $3,53 a$ & $3,25 b^{*}$ & $0,68 c$ \\
\hline & $\begin{array}{c}\text { Prairie } \\
\text { diversifiée }\end{array}$ & $\begin{array}{c}65 \\
(0) / 0 / 21 \\
\end{array}$ & - & - & 3,6 & $18,2 \mathrm{a}$ & $51,7 b$ & 55,7 & 33,2 & $4,63 b$ & $1,19 b$ & $1,30 b$ & $4,57 a^{*}$ & $1,32 a$ \\
\hline \multirow{4}{*}{$\begin{array}{c}\text { Winttington } \\
\text { et al } \\
2006\end{array}$} & $\begin{array}{c}\text { Prairie } \\
\text { temporaire }\end{array}$ & $\begin{array}{c}\text { Au moins } \\
80 \% \\
\text { de RGA }\end{array}$ & 10 & - & - & - & - & 41,9 & 40,6 & $5,6 a$ & $2,6 a^{* *}$ & $1,4^{* *}$ & - & - \\
\hline & Marécage salé & - & 31 & - & - & - & - & 42,6 & 39,8 & $7,3 a$ & $3,1 a^{\star *}$ & $1,6^{* *}$ & - & - \\
\hline & $\begin{array}{l}\text { Lande } \\
\text { à bruyère }\end{array}$ & - & 51 & - & - & - & - & 42,6 & 37,9 & $9,2 b$ & $3,9 b^{* *}$ & $1,7^{* *}$ & - & - \\
\hline & Lande & - & 60 & - & - & - & - & 40,2 & 39,8 & $8,3 b$ & $3,1 a^{* *}$ & $1,7^{\star \star}$ & - & - \\
\hline
\end{tabular}

nes familles botaniques comme les Astéracées et les Apiacées (Collomb et al 2002b). Ces résultats ont été confirmés sur le lait de chèvre par Trana et al (2005) qui ont mis en évidence des corrélations négatives entre la concentration en acide linolénique du lait et le pourcentage de graminées de l'herbe pâturée et des corrélations positives avec le pourcentage en dicotylédones. Ces résultats pourraient traduire l'existence d'inhibiteurs de l'hydrogénation ruminale dus à la présence des métabolites secondaires issus des dicotylédones sans que les causes en soient encore connues. L'effet de l'altitude sur la composition du lait de montagne est souvent significatif : les pâturages d'altitude suisses entraînent une teneur plus élevée en oméga 3 , par rapport à la moyenne montagne ou à la plaine, dans le lait de vache (Bugaud et al 2000, Collomb et al 2002a, 2006, tableau 4) et de brebis (Collomb et al 2006). Les différences de teneur en CLA et en acides gras trans-vaccénique vont dans le même sens et sont souvent plus marquées. Des travaux récents ont également comparé, au cours de la période estivale, des laits produits soit à partir de pâturages d'alpage, soit à partir de pâturages de vallée, avec des rations composées d'herbe et/ou de fourrages conservés : les laits d'alpage étaient en moyenne plus riches en CLA $(+1,3$ $\mathrm{g} / 100 \mathrm{gAGT})$ et en oméga $3(+0,8)$ (Chilliard et al 2007). Les mêmes résultats ont été observés pour les fromages (Zeppa et al 2003). Les augmentations marquées d'oméga 3 et de CLA dans les laits et les fromages d'alpage pourraient être dues à une mobilisation plus poussée des lipides corporels ou à des particularités de la flore alpine réduisant la biohydrogénation ruminale. En zone de plaine en Belgique, la distribution d'ensilage issu d'une prairie permanente diversifiée comparé à un ensilage issu d'une prairie moins diversifiée n'a eu que peu de conséquence sur la teneur en acide linolénique du lait, en revanche, la teneur en CLA a été accrue (Lourenço et al 2005, tableau 4). Les mêmes tendances ont été observées sur prairies permanentes d'Auvergne comparativement à des prairies monospécifiques de Bretagne (Astier et al 2004, tableau 4). Néanmoins, sur deux expérimentations visant plus spécifiquement à mettre en évidence le rôle de la diversité spécifique, les gradients de richesse de ces mêmes acides gras ne sont pas clairs. Sur des prairies normandes (Guichard et al 2006), il semble que ce soit davantage la présence des légumineuses que la richesse en espèces qui expliquent la plus grande teneur en CLA observée. Sur trois prairies permanentes d'Auvergne, le gradient de diversité floristique ne peut être mis en parallèle avec un gradient des teneurs en oméga 3 et en CLA, même si la prairie la plus diversifiée présente les teneurs les plus élevées (Tornambé et al 2007b).

\section{b) Dans les produits carnés}

Pour les produits carnés, Adnoy et al (2005) en Norvège ont observé une teneur plus élevée en acides gras polyinsaturés intramusculaires sur des agneaux pâturant une prairie diversifiée de montagne par rapport à une prairie monospécifique de plaine (tableau 5). En Belgique, la teneur en acides gras polyinsaturés et en acide linolénique du gras sous-cutané a été plus élevée pour des agneaux pâturant des prairies riches en légumineuses comparée à des prairies plus diversifiées ou des prairies riches en graminées (Lourenço et al 2007). Cependant, à l'instar des résultats obtenus sur le lait, les teneurs de la viande en acides gras intermédiaires de la biohydrogénation (CLA en particulier) ont été accrus avec la prairie diversifiée. La viande était en outre plus riche en acides gras à 20 et 22 carbones de la série des n-3 (acides eicosapentaénoïque EPA et docosahexaénoïque DHA). Enfin, Whittington et al (2006) au Royaume-Uni n'ont pas observé de différences de teneur en acides gras du muscle d'agneaux pâturant des prairies à base de ray-grass anglais, des prés salés et deux types de landes. Par contre, le pâturage sur landes a entraîné un accroissement de la teneur des acides gras à 22 carbone (DHA), mais pas de celle des acides gras à 20 carbones (EPA).

De cet ensemble de travaux, il ressort que la teneur en acide linolénique, principal oméga 3 des fourrages à base d'herbe, n'est pas systématiquement plus élevée dans les prairies diversifiées que dans les prairies peu diversifiées ou monospécifiques. L'ingestion 
de fourrages issus de prairies diversifiées n'accroît pas ou augmente modérément la richesse du lait et de la viande en oméga 3 par rapport à des prairies monospécifiques ou des prairies peu diversifiées. En revanche, la composition botanique et en particulier la présence de dicotylédones (ou tout du moins de certaines dicotylédones) semble avoir des conséquences sur le déroulement de la biohydrogénation ruminale des acides gras, sans doute par le biais de métabolites secondaires ou d'enzymes. Ainsi, plusieurs études soulignent l'augmentation de la teneur en acides gras intermédiaires de la biohydrogénation (CLA, acide gras trans) pour les produits laitiers comme pour les produits carnés issus de prairies d'altitude ou de prairies de plaine diversifiées, riches en dicotylédones.

\section{2 / Les autres composés des plantes}

\section{a) Les caroténoïdes}

On regroupe sous le terme de caroténoïdes, les carotènes et les xanthophylles, dont principalement la lutéine, le lycopène et la zéaxanthine. Certains caroténoïdes des plantes ( $\alpha$ - et $\beta$-carotènes principalement) sont les précurseurs de la vitamine A. La vitamine A est une vitamine liposoluble qui est impliquée dans de nombreuses fonctions biologiques, comme le développement embryonnaire, la croissance, la vision et la régulation de l'expression des gènes. Dans la mesure où la plupart des mammifères ne peuvent pas la synthétiser de novo, la vitamine A doit être apportée par l'alimentation. Chez les ruminants, la vitamine A provient principalement de la conversion des carotènes des fourrages en vitamine A mais elle peut également être apportée par les compléments vitaminés distribués aux animaux. Dans l'alimentation humaine, la vitamine A provient directement des produits animaux (foie, viande, produits laitiers et oeufs), alors que les produits végétaux (carottes, fruits, huiles végétales...) fournissent des caroténoïdes dont certaines provitamines A qui peuvent être transformés en vitamine $A$, après un clivage spécifique dans l'intestin ou le foie (Sauvant et al 2002). Les produits laitiers constituent une source intéressante de vitamine $\mathrm{A}$ et de caroténoïdes pour le consommateur adulte et la source principale pour les nouveau-nés (Debry 2001). Il a été également démontré que les caroténoïdes ingérés, indépendamment de leurs effets dus à la vitamine $\mathrm{A}$, jouent un rôle dans les voies de signalisations cellulaires et dans la prévention de plusieurs types de cancers et, potentiellement des maladies cardiovasculaires. Plus spécifiquement, en tant que pigments, la lutéine et la zéaxanthine jouent un rôle dans la vision et permettraient à ce titre de prévenir la dégénérescence maculaire de la rétine liée au vieillissement (Stahl et Sies 2005). Enfin, les caroténoïdes présents dans les produits animaux ont également une activité antioxydante bénéfique pour la santé humaine. Ils sont responsables, en outre, de la coloration jaune des produits laitiers et des tissus adipeux bovins.

L'herbe verte est très riche en ces pigments. Prache et al (2003) font état d'une variation de la concentration totale en caroténoïdes de 430 à 700 $\mathrm{mg} / \mathrm{kg}$ MS au cours de la saison, pour une prairie permanente peu diversifiée (7 espèces végétales, $11 \%$ de dicotylédones). Les concentrations les plus élevées sont observées dans l'herbe verte pâturée au printemps et en automne sur les repousses et sont plus faibles dans l'herbe à un stade de maturité avancée (Calderon et al 2007). Elles sont également réduites fortement après le séchage et la conservation des fourrages (Nozière et al 2006). Sur une prairie permanente peu diversifiée, Graulet et al (2006) ont mesuré une concentration de $741 \mathrm{mg} / \mathrm{kg} \mathrm{MS}$ et identifié au total 8 caroténoïdes, dont $76 \%$ étaient des xanthophylles et $24 \%$ des carotènes. Le taux de transfert des caroténoïdes de la ration au lait est très faible : moins de $1 \%$ des caroténoïdes ingérés sont sécrétés dans le lait (Calderon et al 2007). Pour le lait, si il existe une relation étroite entre la quantité de $\beta$-carotène ingérée et la quantité sécrétée dans le lait, la relation est beaucoup plus lâche voire quasi inexistante pour les xanthophylles (Nozière et al 2006). Pour les produits carnés, en revanche, la loi de réponse entre le niveau de xanthophylles ingérés et leur concentration dans le tissu adipeux (et par conséquence l'indice de jaune de celui-ci) est linéaire (Dian et al 2007). La compilation des données fragmentaires existantes sur les caroténoïdes n'a pas permis de montrer une différence de concentration entre des laits issus de l'apport de foin de prairies monospécifiques ou de prairies permanentes (Nozière et al 2006). Mc Dowall et Mc Gillivray (1963) ont toutefois observé une influence très modérée de la composition botanique de l'herbe : les laits issus des prairies les plus diversifiées avaient tendance à être légèrement moins riches en $\beta$-carotène et vitamine
A que les laits issus de prairies composées uniquement de graminées. Lucas et al 2006 ont également souligné une plus faible concentration en $\beta$-carotène et en vitamine A des fromages produits en altitude lorsque les animaux utilisent des prairies très diversifiées par comparaison aux fromages de plaine issus de pâturages moins diversifiés. Une comparaison sur des laits de mélange a cependant montré un résultat inverse : la teneur en lutéine du lait était plus élevée avec des prairies permanentes auvergnates qu'avec des prairies monospécifiques (Astier et al 2004). L'ensemble de ces résultats ne permet pas de conclure quant à un effet du type de prairies, trop de données manquant pour leur interprétation rigoureuse (stade de l'herbe, composition botanique, type de rations et complémentation vitaminée des animaux).

\section{b) Les polyphénols}

L'Homme, comme les animaux d'élevage, a à sa disposition différentes sources d'antioxydants pour prévenir et/ou limiter les phénomènes oxydatifs (voir $\S 6.2$ ). Ces antioxydants sont d'origine endogène ou nutritionnelle. Les polyphénols contenus dans les produits animaux et provenant des fourrages ingérés peuvent ainsi constituer des apports bénéfiques pour la santé humaine. Comme il a été souligné en première partie, les structures phénoliques sont souvent spécifiques de l'espèce végétale, de la famille botanique ou des conditions environnementales, ce qui explique l'existence d'une très grande quantité et diversité de molécules. Celles-ci peuvent être classées en 4 grands groupes (acides phénoliques, lignanes, stilbènes et flavonoïdes) dont les flavonoïdes représentent le groupe prépondérant.

Plusieurs études soulignent une plus forte concentration en polyphénols des dicotylédones comparativement aux graminées (Scehovic 1990, Jeangros et al 1999), ainsi que l'influence du stade phénologique des plantes. Fraisse et al (2007) ont ainsi mis en évidence la très grande richesse en polyphénols d'une prairie de montagne très diversifiée (43 espèces, $58 \%$ de dicotylédones) : 170 composés phénoliques différents ont été identifiés, parmi lesquels 30 seulement étaient communs à l'ensemble des espèces végétales. Une autre expérimentation comparant différentes rations hivernales n'a cependant pas montré de différences de concentration en polyphénols totaux et en flavonoïdes dans les laits issus de foin de prairie permanente ou de foin de ray-grass 
(Besle et al 2004). Toutefois dans cet essai, la prairie permanente avait été exploitée à un stade très jeune (foin séché en grange) et l'abondance des dicotylédones était vraisemblablement encore faible.

Les données disponibles concernant les caroténoïdes et les polyphénols sont aujourd'hui trop partielles et trop fragmentaires pour conclure quant à l'influence de la composition botanique et de la diversité floristique sur les teneurs en caroténoïdes des fourrages. Des recherches complémentaires sont donc nécessaires. Le rôle probablement important des dicotylédones, plus riches en polyphénols que les graminées, reste à mettre en évidence.

\section{6 / Diversité floristique et santé animale}

Le sujet des liens entre la flore des prairies et la santé animale étant vaste, complexe et encore peu exploré, nous avons choisi de nous centrer sur deux thématiques pour lesquelles nous disposons de connaissances ou d'hypothèses. Nous nous sommes limités aux propriétés antiparasitaires des tannins ainsi qu'aux effets des composés antioxydants présents dans les végétaux sur la prévention des processus de peroxydation. L'intérêt pour les propriétés anthelminthiques des tannins est récent et la littérature scientifique en la matière est importante et d'origine géographique variée. Ce sujet a fait l'objet d'une synthèse récente (Hoste et al 2006). Les travaux concernant les effets anti-oxydants bénéfiques pour les animaux de certains composés végétaux sont beaucoup plus rares. Dans ce domaine, les relations entre les stress oxydants et la santé des animaux d'élevage sont bien établies et ont fait l'objet d'une synthèse relativement récente (Aurousseau 2002). Les mécanismes d'action des différents composés antioxydants sont également relativement bien connus. En revanche il n'y a pas eu d'expérimentations sur animaux démontrant les effets spécifiques des composants antioxydants végétaux et, en particulier ceux présents dans les plantes des prairies permanentes.

\section{1 / Tannins et propriétés anti- parasitaires}

Au delà de leurs effets favorables sur la dégradabilité des protéines dans le rumen, sur leur propriétés antioxydantes et sur leur effet sur les caractéristiques sensorielles traités dans les para- graphes précédents, un intérêt récent s'est manifesté pour les tannins vis-àvis de leurs propriétés antiparasitaires contre les strongles digestifs (Hoste et al 2006). Ces nématodes parasites, très largement répandus chez les ruminants élevés à l'herbe, sont responsables de pertes économiques majeures. Leur maîtrise usuelle, fondée sur l'emploi quasi exclusif d'anthelminthiques de synthèse, est désormais remise en cause par le développement croissant de résistances à ces molécules dans les populations de vers (Waller et al 2006). Tout moyen de lutte innovant offrant une alternative aux anthelminthiques chimiques est par conséquent digne d'intérêt.

L'essentiel des données disponibles sur les propriétés anthelminthiques des tannins concernent certaines légumineuses fourragères telles le lotier corniculé, le lotier pédonculé, le sulla, le sainfoin ou le sericea, riches en tannins condensés, c'est-à-dire des tannins ne traversant pas la barrière intestinale et donc moins toxiques que les tannins hydrolysables, qui peuvent entraîner des phénomènes de nephrotoxicité ou d'hépatotoxicité. Peu de travaux en milieux tempérés se sont intéressés à la présence de tannins dans d'autres plantes fourragères que les légumineuses et globalement dans la prairie permanente. Toutefois, des teneurs importantes en tannins ont été mentionnées dans certaines dicotylédones bien représentées dans les prairies permanentes, comme l'alchémille, le pissenlit et l'achillée millefeuille (Scehovic 1990). La relation avec des propriétés antiparasitaires n'a toutefois pas été vérifiée. Plusieurs études ont également associé des effets anthelminthiques à la consommation de chicorée (Marley et al 2003) mais le rôle des tannins dans l'activité anthelminthique paraît négligeable pour cette plante et pourrait plutôt être liée aux lactones sesquiterpéniques (Hoste et al 2006). A côté de ces études centrées sur les plantes prairiales, d'autres travaux indiquent des propriétés similaires chez des espèces botaniques variées constitutives de maquis, garrigues ou sous bois, ce qui ajoute un intérêt potentiel à l'exploitation pastorale. Plusieurs études ont ainsi souligné les effets bénéfiques associés à la consommation de bruyère chez des chèvres (Osoro et al 2007) ainsi que chez des cerfs et des chèvres parasités pâturant une végétation riche en tannins (Hoste et al 2007). D'autre part, sur la base de résultats in vitro, une assez large gamme d'espèces ligneuses ont été identifiées (chêne, mûrier sauvage, noisetier, genet à balai, bruyère, pin sylvestre, châtaigner) comme dotées de propriétés anthelminthiques (Paolini et al 2004, Bahuaud et al 2006).

La présence d'une activité anthelminthique des tannins sur les principaux nématodes, parasites de l'abomasum (Haemonchus contortus, Teladorsagia circumcincta) ou de l'intestin grêle (Trichostrongylus colubriformis, $\mathrm{Ne}$ matodirus battus) a été vérifiée par de nombreuses études, à l'aide de tests in vitro ou d'essais in vivo en conditions d'infestations expérimentales sur mouton ou chèvre (Athanasiadou et al 2001, Niezen et al 2002, Hoste et al 2006, Shaik et al 2006). De plus, lors des tests in vitro, l'emploi d'inhibiteurs de tannins a permis de confirmer le rôle central joué par ces composés dans l'activité anthelminthique. Chez l'animal, la consommation de ces fourrages a été associée à deux impacts principaux pouvant affecter la dynamique des infestations parasitaires : d'une part, une moindre quantité de vers chez l'hôte, en raison d'une moindre installation des larves infestantes ou d'un effet anthelminthique sur les vers adultes et, d'autre part, une moindre contamination du milieu extérieur, liée à une baisse de ponte des vers femelles. L'efficacité des tannins n'est jamais absolue, les baisses maximales d'excrétion d'œufs signalées atteignant 60 à $70 \%$ (Heckendorn et al 2006). Ces conséquences sur les infestations ont généralement été associées à une meilleure résilience de l'hôte face au parasitisme, s'exprimant par une atténuation des signes cliniques (diarrhée, anémie), des perturbations physiopathologiques ou des pertes de production (Niezen et al 1995, Hoste et al 2005, Paolini et al 2005). Plusieurs questions restent toutefois à résoudre. En premier lieu, il faut comprendre le rôle respectif de facteurs à l'origine d'une variabilité des résultats observés. En effet, selon les études, de fortes variations de propriétés antiparasitaires ont été constatées puisque parfois la consommation de légumineuses riches en tannins par des animaux infestés ne s'est accompagnée d'aucun effet anthelminthique significatif. Des facteurs liés à la plante, à l'hôte ou aux parasites (espèces, stade) peuvent expliquer de telles variations. Pour ceux liés à la plante, il semble qu'une concentration minimale de tannins condensés dans la ration soit nécessaire pour obtenir des effets antiparasitaires. Les données actuelles suggèrent que ce seuil se situerait autour de $3 \%$ de la MS de la ration (Min et Hart 
2002, Hoste et al 2006). Ce sont des teneurs très élevées, que l'on a peu de chance d'obtenir sur des couverts plurispécifiques. D'autre part, la nature des tannins intervient également, les propriétés antiparasitaires étant associées essentiellement aux tannins condensés. Certaines données suggèrent que l'activité anthelminthique serait d'abord liée à la présence d'une classe particulière de tannins condensés : les prodelphinidines (Brunet et Hoste 2006). La variabilité des effets liée à l'espèce parasite est surtout le constat d'études in vivo alors que les mesures in vitro n'indiquent que des différences spécifiques mineures. Enfin, en dépit de certaines avancées récentes, les mécanismes d'action des tannins sur les divers stades de nématodes restent encore mal connus.

Ainsi, de nombreuses questions sur les modes d'actions des tannins sur les vers restent à élucider, mais les résultats obtenus sur certaines légumineuses sont encourageants et illustrent des propriétés anthelminthiques longtemps ignorées. Ils engagent à étendre cette approche à d'autres espèces végétales présentes dans les prairies permanentes et à s'intéresser plus globalement aux teneurs présentes dans ces dernières. Par ailleurs, des travaux récents mettent en évidence des propriétés similaires sur des espèces ligneuses fréquemment rencontrées sur les parcours.

\section{2 / Antioxydants végétaux et prévention des processus de per- oxydation}

Le stress oxydant résulte d'une situation où apparaît un déséquilibre entre les substances antioxydantes et la production d'Espèces Radicalaires Oxygénées (ERO) qui sont particulièrement agressives vis-à-vis de l'organisme. Différentes conditions d'élevage peuvent conduire à une surproduction d'ERO qui dépasse largement la capacité antioxydante de l'organisme (Aurousseau 2002). Ce n'est qu'à partir des années 1985-1990 que des relations entre l'augmentation des processus de peroxydation et la santé animale ont été réellement étudiées. Une première relation a été établie entre le stress oxydant et la capacité de reproduction (Takayanagi et al 1986). D'autres études ont ensuite montré que les nombreux problèmes sanitaires observés en peripartum (rétention placentaire, œdème du pis, mammite, métrite, infertilité, fièvre de lait) étaient toujours associés à une augmentation des phénomènes radicalaires (Miller et al
1993). Ces études ont permis de mettre en évidence que la maîtrise des processus oxydatifs au cours de la vie des animaux est essentielle pour assurer des conditions d'élevage et de production optimales, d'autant plus que les processus oxydatifs générés au niveau de l'animal lui-même peuvent avoir des conséquences très négatives comme l'apparition de défauts de flaveur sur les produits issus de ces animaux (Durand et al 2005). Dans un tel contexte, les défenses antioxydantes de l'animal jouent un rôle essentiel et l'apport d'antioxydants exogènes dits «naturels» par le biais de l'alimentation est devenu un enjeu essentiel pour les nouvelles stratégies de conduites des animaux et pour la recherche en santé animale. Les études sur la quantité et la qualité des antioxydants naturels sont relativement récentes en particulier chez le monogastrique (volaille principalement), mais également chez le ruminant (Durand et al 2005, Gladine et al 2007). Elles montrent que les antioxydants sont des composés très diversifiés, qui à faible concentration par rapport à celle du substrat oxydable, retardent ou inhibent significativement l'oxydation de ce substrat (Halliwell 1990). De très nombreuses familles de composés présents dans les végétaux ont un pouvoir antioxydant. Les caroténoïdes, les polyphénols, la vitamine $\mathrm{E}$, la vitamine $C$ et l'acide phytique constituent l'essentiel de ces composés (Di et al 1990, Morel et al 1993, Frankel 1998, May 2000, Vertuani et Manfredi 2004). Leurs localisations et leurs niveaux d'action dans l'organisme sont multiples. Ils sont localisés à la fois dans les phases aqueuses et membranaires et à des niveaux différents depuis les compartiments digestifs jusque dans le lait et les muscles. Ils exercent leur action antioxydante à différents niveaux de la chaîne de peroxydation. Selon leurs mécanismes d'action et leur niveau d'intervention dans la chaîne de peroxydation, ils peuvent être classés en deux groupes, l'un dits «préventifs» : ils empêchent la formation d'ERO, l'autre dit "casseurs de châ̂ne» : ils interceptent les espèces radicalaires secondaires formées à partir des substrats oxydables. Cette complémentarité d'action des composés antioxydants suggère l'importance de fournir aux animaux des sources d'alimentation diversifiées. Dans ce cadre, nous ne pouvons aujourd'hui que poser l'hypothèse selon laquelle la prairie permanente diversifiée riche en composés secondaires constituerait une source de composés antioxydants de nature très diverse qui pourrait augmenter la capa- cité antioxydante globale des animaux via les complémentarités d'actions mis en jeu.

\section{Conclusion}

Ce large panorama de l'intérêt de la diversité floristique des prairies permanentes pour la valeur alimentaire des fourrages, la qualité des produits animaux, et deux aspects de santé animale constitue un premier état des connaissances pluridisciplinaires en la matière. Il s'intègre dans la problématique plus vaste des services rendus par la biodiversité à l'agriculture. Dans chacune des thématiques abordées, les effets de la composition botanique des prairies permanentes ont été mis en évidence mais sans que les effets liés spécifiquement à la diversité soient clairement démontrés. En effet, nous n'avons pas été en mesure de faire la part des effets liés à la présence de composés spécifiques issus d'une espèce ou d'une famille d'espèces particulières, des effets liés aux stades phénologiques, de ceux liés au nombre d'espèces. Le rôle de l'identité spécifique a été souligné à maintes reprises en pointant dans chacun des domaines, le rôle probable joué par les dicotylédones et souvent les légumineuses. Nous avons par exemple très souvent montré ou bien envisagé l'action de métabolites secondaires, plus présents dans les dicotylédones que dans les graminées. Nous avons par ailleurs souligné que les connaissances sur les mécanismes mis en jeu restent dans l'ensemble des domaines à préciser ou à élucider. Nous avons également montré, notamment au travers de l'examen des thématiques liées à l'ingestion et à la santé animale, l'intérêt de fournir aux animaux des sources d'alimentation variées. Cette diversification permet par exemple de multiplier les possibilités de complémentarité d'actions des composés d'origine végétale bénéfiques pour la santé animale. Enfin, l'examen successif des différentes thématiques a mis en évidence que certains métabolites peuvent être responsables de plusieurs effets. Les tannins ingérés en sont l'exemple le plus frappant. Ils peuvent en effet, intervenir dans les processus de dégradabilité des protéines dans le rumen, exercer une activité anthelminthique et antioxydante et avoir un impact sur les caractéristiques sensorielles et nutritionnelles des produits animaux. Pour terminer, notons que nous nous sommes centrés dans cette synthèse sur l'intérêt de la diversité floristique à l'échelle de l'animal et de ses produits. 
Des travaux récents abordent cette thématique sur un plan plus agronomique et à l'échelle de l'exploitation d'élevage. Ils mettent en évidence l'effet positif de la diversité floristique sur la pro- duction de biomasse et démontrent qu'une diversité, non plus intra parcellaire, mais inter parcellaire au sein de l'exploitation est susceptible de constituer un atout pour sécuriser le système fourrager et donner de la flexibilité à la gestion des ressources fourragères (Andrieu et al 2007).

\section{Références}

Adnoy T., Haug A., Sorheim O., Thomassen M.S., Varszegi Z., Eik L.O., 2005. Grazing on mountain pastures - does it affect meat quality in lambs? Livest. Prod. Sci., 94, 25-31.

Agreil C., Fritz H., Meuret M., 2005. Maintenance of daily intake through bite mass diversity adjustment in sheep grazing on heterogeneous and variable vegetation. Appl. Anim. Behav. Sci., 91, 35-56.

Al Haj Khaled R., Duru M., Decruyenaere V., Cruz P., 2006. Using leaf traits to rank native grasses according to their nutritive value. Rangeland Ecol. Manag., 59, 548-654.

Andrieu J., Demarquilly C., Wegat-Litre E., 1981. Prévision de la valeur nutritive des aliments de ruminants. Tables de prévision de la valeur alimentaire des fourrages. INRA (Ed.), Versailles, France, 580p.

Andrieu N., Poix C., Josien E., Duru M., 2007. Simulation of forage management strategies considering farm-level land diversity: Example of dairy farms in the Auvergne. Comp. Elect. Agric., 55, 36-48.

Ansquer P., 2006. Caractérisation agroécologique des végétations prairiales naturelles en réponse aux pratiques agricoles. Apports pour la construction d'outils de diagnostic. Thèse de docteur de l'Institut National Polytechnique de Toulouse, France, 272p.

Ansquer P., Theau J.P., Cruz P., Viegas J., Al Haj Khaled R., Duru M., 2004. Caractérisation de la diversité fonctionnelle des prairies naturelles. Une étape vers la construction d'outils pour gérer les milieux à flore complexe. Fourrages, 178, 353-368.

Astier C., Rock E., Chardigny J.M., Borel P., Coulon J.B., 2004. Effet de l'origine géographique et du traitement thermique du lait de vache sur sa qualité nutritionnelle. Renc. Rech. Rum., 11, 75 .

Athanasiadou S., Kyriazakis I., Jackson F., Coop R.L., 2001. Direct anthelmintic effects of condensed tannins towards different gastrointestinal nematodes of sheep: in vitro and in vivo studies. Vet. Parasitol., 99, 205-219.

Aufrere J., Dudilieu M., Poncet C., Baumont R., 2005. Effect of condensed tannins in sainfoin on in vitro protein solubility of lucerne. XX Int. Grassl. Cong., O'Hara, F. P., Wilkins, R. J., t'Mannetje, L., Lovett, D. K., Rogers, P. A. M., Boland, T. M. (Eds.), Wageningen Academic Publishers, Dublin, Ireland, 248

Aufrere J., Carrere P., Dudilieu M., Baumont R., 2008. Estimation of nutritive value of grasses from semi-natural grasslands, by biological, chemical and enzymatic methods. $22^{\text {th }}$ Gen. Meet. Eur. Grassland Fed., Uppsala, Suède, sous presse.

Aurousseau B., 2002. Les radicaux libres dans l'organisme des animaux d'élevage : conséquences pour la reproduction, la physiologie et la qualité de leurs produits. INRA Prod. Anim., 15, 67-82.
Bahuaud D., De Montellano C.M.O., Chauveau S., Prevot F., Torres-Acosta F., Fouraste I., Hoste H., 2006. Effects of four tanniferous plant extracts on the in vitro exsheathment of third-stage larvae of parasitic nematodes. Parasitology, 132, 545-554

Balent G., Alard D., Blanfort V., Gibon A., 1998. Activités de pâturage, paysages et biodiversité. Ann. Zootech., 47, 419-429.

Bauchart D., Roy A., Lorenz S., Ferlay A., Gruffat D., Chardigny J.M., Sébédio J.L., Chilliard Y., Durand D., 2007. Dietary supply of butter rich in trans 18:1 isomers or in 9cis, 11trans conjugated linoleic acid affects plasma lipoproteins in hypercholesterolemic rabbits. Lipids, 42, 123-133.

Bauman D.E., Corl B.A., Baumgard L.H., Griinari J.M., 2001. Conjugated linoleic acid (CLA) and the dairy cows. In: Recent advances in animal nutrition, Nottingham University Press, Nottingham, UK, 221-250.

Baumont R., Pomiès D., 2004. Feed preferences and voluntary intake of dairy heifers fed grass silage and hays offered singly or as a matter of choice. 20 ${ }^{\text {th }}$ Gen. Meet. Eur. Grassland Fed., Luzern, Switzerland, 1089-1091.

Baumont R., Dulphy J.P., Sauvant D., Meschy F. Aufrère J., Peyraud J.L., 2007. Valeur alimentaire des fourrages et des matières premières : tables et prévision. In : Alimentation des bovins, ovins et caprins. Besoins des animaux. Valeurs des aliments. Tables INRA 2007, Editions Quae, Paris, France, 149-179.

Besle J.M., Lamaison J.L., Pradel P., Fraisse D., Viala D., Martin B., 2004. Les flavonoïdes, des fourrages au lait. Renc. Rech. Rum., 11, 67.

Bruinenberg M.H., Valk H., Korevaar H., Struik P.C., 2002. Factors affecting digestibility of temperate forages from seminatural grasslands: a review. Grass Forage Sci., 57, 292-301.

Bruinenberg M.J., Valk H., Struit P.C., 2003. Voluntary intake and in vivo digestibility of forages from semi-natural grasslands in dairy cows. Neth. J. Agric. Sci., 51, 235.

Brunet S., Hoste H., 2006. Monomers of condensed tannins affect the larval exsheathment of parasitic nematodes of ruminants. J. Agric. Food Chem., 54, 7481-7487.

Buchin S., Martin B., Dupont D., Bornard A., Achilleos C., 1999. Influence of the composition of alpine highland pasture on the chemical, rheological and sensory properties of cheese. $\mathrm{J}$. Dairy Res., 66, 579-588.

Bugaud C., Doreau M., Chabrot J., Hauwuy A., Buchin S., 2000. Composition en acides gras des laits alpins. Relation avec la composition en acides gras des pâtures. In : 11 ème Réunion du sous-réseau Pâturage de montagnes, FAOCIHEAM, Luz-Saint-Sauveur, France, 3p.

Bugaud C., Buchin S., Coulon J.B., Hauwuy A., Dupont D., 2001. Influence of the nature of alpine pastures on plasmin activity, fatty acid and volatile compound composition of milk. Lait $81,401-414$.

Bugaud C., Buchin S., Hauwuy A., Coulon J.B., 2002. Texture et flaveur du fromage selon la nature du pâturage : cas du fromage d'Abondance. INRA Prod. Anim., 15, 31-36.

Calderon F., Chauveau-Duriot B., Martin B., Graulet B., Doreau M., Noziere P., 2007. Variations in carotenoids, vitamins A and $\mathrm{E}$, and color in cow's plasma and milk during late pregnancy and the first three months of lactation. J. Dairy Sci., 90, 2335-2346.

Champion R.A., Orr R.J., Penning P.D., Rutter S.M. 2004. The effect of the spatial scale of heterogeneity of two herbage species on the grazing behaviour of lactating sheep. Appl. Anim. Behav. Sci., 88, 61-76.

Chardigny J.M., 2007. Trans fatty acids from partially hydrogenated oils banned from New York restaurants. What about ruminant trans fatty acids? Eur. J. Lipid Sci. Technol., 109, 645-646.

Chilliard Y., Ferlay A., Mansbridge R.M., Doreau M., 2000. Ruminant milk fat plasticity: nutritional control of saturated, polyunsaturated, trans and conjugated fatty acids. Ann. Zootech., $49,181-205$.

Chilliard Y., Ferlay A., Doreau M., 2001. Effect of different types of forages, animal fat or marine oils in cow's diet on milk fat secretion and composition, especially conjugated linoleic acid (CLA) and polyunsaturated fatty acids. Livest. Prod. Sci., 70, 31-48.

Chilliard Y., Glasser F., Ferlay A., Bernard L., Rouel J., Doreau M., 2007. Diet, rumen biohydrogenation and nutritional quality of cow and goat milk fat. Eur. J. Lipid Sci. Technol., 109, 828-855.

Chilliard Y., Bauchart D., Lessire M., Schmidely P., Mourot J., 2008. Qualité des produits : modulation par l'alimentation des animaux de la composition en acides gras du lait et de la viande. In : Numéro spécial Anniversaire «20 ans de recherche en productions animales à l'INRA». INRA Prod. Anim., 21, 95-106.

Clapham W.M., Foster J.G., Neel J.P.S., Fedders J.M., 2005. Fatty acid composition of traditional and novel forages. J. Agric. Food Chem., 53, 10068-10073.

Clergue B., Amiaud B., Pervanchon F., Lasserre-Joulin F., Plantureux S., 2005. Biodiversity: function and assessment in agricultural areas. A review. Agronomy for Sustainable Development, 25, 1-15.

Collomb M., Butikofer U., Spahni M. Jeangros B., Bosset J.O., 1999. Fatty acid and glyceride composition of cow's milk fat in highand lowland regions. Sciences des Aliments, 19, 97-110.

Collomb M., Butikofer U., Sieber R., Jeangros B., Bosset J.O., 2002a. Composition of fatty acids in cow's milk fat produced in the lowlands, mountains and highlands of Switzerland using high-resolution gas chromatography. Int. Dairy J., 12, 649-659. 
Collomb M., Butikofer U., Sieber R., Jeangros B., Bosset J.O., 2002b. Correlation between fatty acids in cows' milk fat produced in the Lowlands, Mountains and Highlands of Switzerland and botanical composition of the fodder. Int. Dairy J. 12, 661-666.

Collomb M., Butikofer U., Maurer J., Sieber R., 2006. Composition en acides gras du lait de brebis produit à diverses altitudes. Rev. Suisse Agric., 38, 335-339.

Côrtes C., Damasceno J.C., Jamot J., Prache S., 2006. Ewes increase their intake when offered a choice of herbage species at pasture. Anim. Sci., 82, 183-191.

Coulon J.B., Priolo A., 2002. La qualité sensorielle des produits laitiers et de la viande dépend des fourrages consommés par les animaux. INRA Prod. Anim., 15, 333-342.

Coulon J.B., Delacroix-Buchet A., Martin B., Pirisi A., 2005. Facteurs de production et qualité sensorielle des fromages. INRA Prod. Anim., 18, 49-62.

Cruz P Duru M., Therond O., Theau J.P., Ducourtieux C., Jouany C., Al Haj Khaled R., Ansquer P., 2002b. Une nouvelle approche pour caractériser les prairies naturelles et leur valeur d'usage. Fourrages, 172, 335-354.

Daccord R., Wyss U., Kessler J., Arrigo Y., Rouel M., Lehman J., Jeangros B., 2006. Valeur nutritive des fourrages, In :Apports alimentaires recommandés et tables de la valeur nutritive des aliments pour les ruminants. Valeur nutritive des fourrages, On line publishing, Station de recherche Agroscope Liebefeld-Posieux, Posieux, chap. $13,18 \mathrm{p}$.

Debry G., 2001. Lait, nutrition et santé. Tec \& Doc (Ed), Paris, France, 566p

De La Torre A., Debiton E., Durand D., Chardigny JM., Berdeaux O., Barthomeuf C., Bauchart D., Gruffat D., 2005. Conjugated linoleic acid isomers and their conjugated derivatives inhibit growth of human cancer cell lines. Anticancer Res., 25, 3943-3950.

De La Torre A., Debiton E., Durand D., Juanéda P., Durand D., Chardigny J.M., Barthomeuf C. Bauchart D., Gruffat D., 2006. Beef conjugated linoleic acid isomers reduce human cancer cell growth even when associated to other beef fatty acids. Br. J. Nutr., 95, 346-352.

Di M.P., Devasagayam T.P., Kaiser S., Sies H., 1990. Carotenoids, tocopherols and thiols as biological singlet molecular oxygen quenchers. Biochem. Soc. Trans., 18, 1054-1056.

Dian P.H.M., Chauveau-Duriot B., Prado I.N., Prache S., 2007. A dose-response study relating the concentration of carotenoid pigments in blood and reflectance spectrum characteristics of fat to carotenoid intake level in sheep. J. Anim. Sci., 85, 3054-3061.

Doreau M., Lee M.R.F., Ueda K., Scollan N.D., 2005. Métabolisme ruminal et digestibilité des acides gras des fourrages. Renc. Rech. Rum., 12, 101.

Duncan A.J., Ginane C., Gordon I.J., Orskov E.R., 2003. Why do herbivores select mixed diets? VIth Int. Symp. Nutr. Herbivores, t'Mannetje, L (Ed), Merida, Mexico, 209.

Durand D., Scislowski V., Gruffat D., Chilliard Y., Bauchart D., 2005. High-fat rations and lipids peroxydation in ruminants: consequences on the health of animals and quality of their products. In: Indicators of milk and beef quality, Hocquette J.F., Gigli S. (Eds), Wageningen Academic Publishers, Wageningen, Netherland, 137-148.
Duru M., 1997. Leaf and stem in vitro digestibility for grasses and dicotyledons of meadow plant communities in spring. J. Sci. Food Agric., 74, 175-185.

Duru M., Cruz P., Magda D., 2004. Using plant traits to compare sward structure and composition of grass species across environmental gradients. Appl.Veget. Sci., 7, 11-18.

Fiems L.O., De Boever J.L., De Vliegher A., Vanacker J.M., De Brabander D.L., Carlier L., 2004. Agri-environmental grass hay: nutritive value and intake in comparison with hay from intensively managed grassland. Arch. Anim. Nutr., Arch.Tierernahrung, 58, 233-244.

Fleury P., Jeannin B., Dorioz J.M., 1990 Quelle méthode choisir pour décrire la diversité de valeur nutritive des fourrages dans les prairies permanentes? Exemple des prairies de fauche des Alpes du Nord françaises. Herba, 3, 36-39.

Fraisse D., Carnat A., Viala D., Pradel P., Besle J.M., Coulon J.B., Felgines, C., Lamaison, J.L., 2007. Polyphenolic composition of a permanent pasture: Variations related to the period of harvesting. J. Sci. Food Agric., 87, 24272435.

Frankel E.N., 1998. Lipid oxidation. $1^{\text {st }}$ edition, Theo il Press Ltd, Dundee, Scotland, 23-78.

Garnier, E., Shipley, B., Roumet, C., Laurent, G., 2001. A standardized protocol for the determination of specific leaf area and leaf dry matter content. Funct. Ecol., 15, 688-695.

Gibon A., Balent G., Duru M., Magda J.P., Theau J.P., Calvière I., Sos L., 1997. Les prairies permanentes de milieu océanique et de montagne humide. Outils de diagnostic agro-climatique et guide pour leur utilisation. INRA (Ed.), Toulouse, France, 51p.

Ginane C., Baumont R., Lassalas J., Petit, M., 2002. Feeding behaviour and intake of heifers fed on hays of various quality, offered alone or in a choice situation. Anim. Res., 51, 177-188.

Gladine C., Morand C., Rock E., Bauchart D., Durand D., 2007. Plant extracts rich in polyphenols (PERP) are efficient antioxidants to prevent lipoperoxidation in plasma lipids from animals fed n - 3 PUFA supplemented diets. Anim. Fd Sci. Techn., 136, 281-296.

Glasser F. Schmidely P., Sauvant D., Doreau M., 2008. Digestion of fatty acids in ruminants: a meta-analysis of flows and variation factors: 2 . C18 fatty acids. Animal, 2, 691-704.

Grant W.B., 1998. Milk and other dietary influences on coronary heart disease. Altern. Med. Rev., 3, 281-294.

Graulet B., Chauveau-Duriot B., Noziere P., Doreau M., 2006. Teneur et composition en caroténoïdes des contenus digestifs et du plasma chez la vache au pâturage. Renc. Rech. Rum., 13, 193.

Griinari J.M., Corl B.A., Lacy S.H., Chouinard P.Y., Nurmela K.V.V., Bauman D.E., 2000. Conjugated linoleic acid is synthesized endogenously in lactating dairy cows by D9desaturase. J. Nutr., 130, 2285-2291.

Grime J.P., Hodgson J.G., Hunt R., 1988. Comparative plant ecology - A functional approach to common British species, Academic Division of Unwin Hyman Ldt (Ed), UK, $741 \mathrm{p}$.

Guichard H., Leconte D., Picoche B., Pages J., Simon J.C., 2006. Influence de la composition floristique des prairies permanentes normandes sur les caractéristiques des laits crus dérivés. Fourrages, 188, 457-475.
Guo Q F., 2007. The diversity-biomass-productivity relationships in grassland management and restoration. Basic Appl. Ecol., 8, 199-208.

Halliwell B., 1990. How to characterize a biological antioxidant. Free Radic. Res. Commun., 9, 1-32.

Heckendorn F., Haring D.A., Maurer V., Zinsstag J., Langhans W., Hertzberg H., 2006. Effect of sainfoin (Onobrychis viciifolia) silage and hay on established populations of Haemonchus contortus and Cooperia curticei in lambs. Vet. Parasitol., 142, 293-300.

Hector A, Schmid B, Beierkuhnlein C, Caldeira M C, Diemer M, Dimitrakopoulos P G, Finn J A, Freitas H, Giller P S, Good J, Harris R, Hogberg P, Huss-Danell K, Joshi J, Jumpponen A, Korner C, Leadley P W, Loreau M, Minns A, Mulder C P H, O'Donovan G, Otway S J, Pereira J S, Prinz A, Read D J, Scherer-Lorenzen M, Schulze E D, Siamantziouras A S D, Spehn E M Terry A C, Troumbis A Y, Woodward F I, Yachi S, Lawton J H., 1999. Plant diversity and productivity experiments in European grasslands. Science, 286, 1123-1127.

Hector A., Bagchi R., 2007. Biodiversity and ecosystem multifunctionality. Nature, 448, 188-196.

Hooper D.U., Chapin F.S., Ewel J.J., Hector A., Inchausti P., Lavorel S., Lawton J.H., Lodge D.M., Loreau M., Naeem S., Schmid B., Setala H., Symstad A.J., Vandermeer J., Wardle D.A., 2005. Effects of biodiversity on ecosystem functioning: A consensus of current knowledge. Ecol. Monographs, 75, 3-35.

Hoste H., Gaillard L., Le Frileux Y., 2005. Consequences of the regular distribution of sainfoin hay on gastrointestinal parasitism with nematodes and milk production in dairy goats. Small Rumin. Res., 59, 265-271.

Hoste H., Jackson F., Athanasiadou S., Thamsborg S.M., Hoskin S.O., 2006. The effects of tannin-rich plants on parasitic nematodes in ruminants. Trends Parasitol., 22, 253-261.

Hoste H., Brunet S., Paolini V., Bahuaud D., Chauveau S., Fouraste I., LeFrileux Y., 2007. Compared in vitro anthelmintic effects of eight tannin-rich plants browsed by goats in the southern part of France. Options Méditerrannénnes, sous presse.

Huyghe C., Litrico I., 2008. Analyse de la relation entre diversité spécifique des prairies et valeur agronomique : synthèse bibliographique. In : Prairies multispécifiques, Actes des journées AFPF, Paris, France, 26-27 mars 2008.

Jarrige R., Grenet E., Demarquilly C., Besle J.M., 1995. Les constituants de l'appareil végétatif des plantes fourragères. In: Nutrition des ruminants domestiques, ingestion et digestion, Jarrige R., Ruckebusch Y., Demarquilly C., Farce M.H., Journet M. (Eds.), INRA Editions, Paris, France, 25-71.

Jeangros B., Scehovic J., Troxler J., Bachmann H.J., Bosset J.O., 1999. Comparaison de caractéristiques botaniques et chimiques d'herbages pâturés en plaine et en montagne. Fourrages 159, 277-292.

Julier B., Lila M., Huyghe C., Morris P., Allison G., Robbins M., 2002. Effect of condensed tannin content on protein solubility in legume forage. Grassland Sci. Europe, 7, 134135.

Julier B., Guines F., Emile J.C., Huyghe C. 2003. Variation in protein degradability in dried forage legumes. Animal Research, 52, 401-412.

Kirwan L, Luescher A, Sebastia M T, Finn J A, Collins R P, Porqueddu C, Helgadottir A, 
Baadshaug $\mathrm{O} \mathrm{H}$, Brophy $\mathrm{C}$, Coran $\mathrm{C}$, Dalmannsdottir S, Delgado I, Elgersma A, Fothergill M, Frankow-Lindberg B E, Golinski P, Grieu P, Gustavsson A M, Hoglind M, Huguenin-Elie $\mathrm{O}$, Iliadis $\mathrm{C}$, Jorgensen $\mathrm{M}$, Kadziuliene Z, Karyotis T, Lunnan T, Malengier M, Maltoni S, Meyer V, Nyfeler D, NykanenKurki P, Parente J, Smit H J, Thumm U, Connolly J., 2007. Evenness drives consistent diversity effects in intensive grassland systems across 28 European sites. J. Ecol., 95, 530-539.

Larick D.K., Hedrick H.B., Bailey M.E., Williams J.E., Hancock D.L., Garner G.B., Morrow R.E., 1987. Flavor constituents of beef as influenced by forage- and grain-feeding. J. Food Sci., 52, 245-251.

Le Gall A., Pflimlin A., Maheas M., 2007. La prairie, un enjeu économique et sociétal. In : Le dossier Economie de l'Elevage, Institut de l'Elevage (Ed), Paris, France, 1-40.

Lee M.R.F., Connelly P.L., Tweed J.K.S., Dewhurst R.J., Merry R.J., Scollan N.D., 2006. Effects of high-sugar ryegrass silage and mixtures with red clover silage on ruminant digestion.

2. Lipids. J. Anim. Sci., 84, 3061-3070.

Loiseau P., 2004. Introduction à l'appréciation de la diversité d'une communauté prairiale. Document de formation, 3-4 juin 2004, INRA Theix.

Louault F., Pillar PVD, Aufrère J., Garnier E., Soussana J.F., 2005. Plant traits and functional types in response to reduced disturbance in a seminatural grassland. J. Veg. Sci., 16, 151-160.

Lourenço M., Vlaeminck B., Bruinenberg M., Demeyer D., Fievez V., 2005. Milk fatty acid composition and associated rumen lipolysis and fatty acid hydrogenation when feeding forages from intensively managed or semi-natural grasslands. Anim. Res., 54, 471-484.

Lourenço M., Van Ranst G., De Smet S., Raes K., Fievez V., 2007. Effect of grazing pasture with different botanical composition by lambs on rumen fatty acid metabolism and fatty acid pattern of longissimus muscle and subcutaneous fat. Animal, 1, 537-545.

Lucas A., Agabriel C., Martin B., Ferlay A., Verdier-Metz I., Coulon J. B., 2006. Relationships between the conditions of cow's milk production and the contents of components of nutritional interest in raw milk farmhouse cheese. Lait, 86, 177-202.

Magurran A.E., 2003. Measuring biological diversity. Blackwell Publishing, Oxford, University of St Andrews, UK, 256p.

Marley C.L., Cook R., Keatinge R., Barrett J., Lampkin N.H., 2003. The effect of birdsfoot trefoil (Lotus corniculatus) and chicory (Cichorium intybus) on parasite intensities and performance of lambs naturally infected with helminth parasites. Vet. Parasitol., 112, 147-155.

Martin B., Verdier-Metz I., Buchin S., Hurtaud C., Coulon J.B., 2005. How do the nature of forages and pasture diversity influence the sensory quality of dairy livestock products? Anim. Sci., 81, 205-212.

May J.M., 2000. How does ascorbic acid prevent endothelial dysfunction? Free Radical Biol Med., 28, 1421-1429.

Mc Dowall F.H. Mc GilLivray A., 1963. Studies on the properties of New Zealand butterfat - VII. Effect of the stage of maturity of ryegrass fed to cows on the characteristics of butterfat and its carotene and vitamin A contents. J. Dairy Res., 30, 59-66.
Merry R.J., Lee M.R.F., Davies D.R., Dewhurst R.J., Moorby J.M., Scollan N.D., Theodorou M.K., 2006. Effects of high-sugar ryegrass silage and mixtures with red clover silage on ruminant digestion. 1 . In vitro and in vivo studies of nitrogen utilization. J. Anim. Sci., 84, 3049-3060.

Meuret M., 1993. Piloter l'ingestion au pâturage. In: Pratiques d'élevage extensif. Identifier, modéliser, évaluer, Landais E. (Ed), Nathan, Paris, France, 161-198.

Meuret M., Bruchou C., 1994. Modélisation de l'ingestion selon la diversité des choix alimentaires réalisés par la chèvre au pâturage sur parcours. Renc. Rech. Rum., 1, 225.

Miller J.K., Brzezinskaslebodzinska E., Madsen F.C., 1993. Oxidative stress, antioxidants, and animal function. J. Dairy Sci., 76, 2812-2823.

Min B.R., Hart S.P., 2002. Tannins for suppression of internal parasites. J.Anim. Sci., 81, 102-109.

Min B.R., Mcnabb W.C., Barry T.N., Peters J.S., 2000. Solubilization and degradation of ribulose-1,5-bisphosphate carboxylase/oxygenase (EC 4.1.1.39; Rubisco) protein from white clover (Trifolium repens) and Lotus corniculatus by rumen microorganisms and the effect of condensed tannins on these processes. J. Agric. Sci., 34, 305-317.

Morel I., Lescoat G., Cogrel P., Sergent O. Pasdeloup N., Brissot P., Cillard P., Cillard J., 1993. Antioxidant and iron-chelating activities of the flavonoids catechin, quercetin and diosmetin on iron-loaded rat hepatocyte cultures. Biochem. Pharmacol., 45, 13-19.

Morel I., Wyss U., Collomb M., Butikofer U., 2006. Influence de la composition botanique de l'herbe ou du foin sur la composition du lait. Rev. Suisse Agric., 38, 9-15.

Niezen J.H., Waghorn T.S., Charleston W.A.G., Waghorn G.C., 1995. Growth and gastrointestinal nematode parasitism in lambs grazing either lucerne (Medicago-Sativa) Or Sulla (Hedysarum-Coronarium) which contains condensed tannins. J. Agric. Sci., 125, 281289.

Niezen J.H., Waghorn G.C., Graham T., Carter J.L., Leathwick D.M., 2002. The effect of diet fed to lambs on subsequent development of Trichostrongylus colubriformis larvae in vitro and on pasture. Vet. Parasitol., 105, 269-283.

Noziere P., Graulet B., Lucas A., Martin B., Grolier P., Doreau M., 2006. Carotenoids for ruminants: from forages to dairy products. Anim. Food Sci. Techn., 131, 418-450.

Nozières M.O., Dulphy J.P., Peyraud J.L., Baumont R., 2006. Prediction of forage rumen protein degradability from an in situ degradability database. 21 $1^{\text {th }}$ Gen. Meet. Grassland Sci. Eur., Lloveras J., Gonzalez-Rodriguez A., Vazquez-Yanez O., Pineiro J., Santamaria O., Olea L., Poblaciones M. J. (Eds) Badajoz, Spain, 531-533.

Nuernberg K., Dannenberger D., Nuernberg G., Ender K., Voigt J., Scollan N.D., Wood J.D., Nute G.R., Richardson R.I., 2005. Effect of a grass-based and a concentrate feeding system on meat quality characteristics and fatty acid composition of longissimus muscle in different cattle breeds. Livest. Prod. Sci., 94, 137-147.

Osoro K., Mateos-Sanz A., Frutos P., Garcia U., Ortega-Mora L.M., Ferreira L.M.M., Celaya R., Ferre, I., 2007. Anthelmintic and nutritional effects of heather supplementation on Cashmere goats grazing perennial ryegrass-white clover pastures. J. Anim. Sci., 85, 861-870.

Paolini V., Fouraste I., Hoste H., 2004. In vitro effects of three woody plant and sainfoin extracts on 3rd-stage larvae and adult worms of three gastrointestinal nematodes. Parasitology, 129 69-77.

Paolini V., De La Farge F., Prevot F., Dorchies $P$, Hoste $H, 2005$. Effects of the repeated distribution of sainfoin hay on the resistance and the resilience of goats naturally infected with gastrointestinal nematodes. Vet. Parasitol., 127 277-283.

Park R.J., 1972. Flavour differences in meat from lambs grazed on lucerne (Medicago sativa) or phalaris (Phalaris tuberosa) pastures. J. Agric. Sci., 78, 47-52.

Plantureux S., 1996. Biodiversity, type of soil and management intensity of permanent pastures of Plateau Lorrain. Acta Botanica Gallica, 143 339-348.

Pontes L.S., Carrere P., Andueza D., Louault F., Soussana J.F., 2007a. Seasonal productivity and nutritive value of temperate grasses found in semi-natural pastures in Europe: responses to cutting frequency and $\mathrm{N}$ supply. Grass Forage Sci., 62, 485-496.

Pontes L.D.S., Soussana J.F., Louault F., Andueza D., Carrere P., 2007b. Leaf traits affect the above-ground productivity and quality of pasture grasses. Funct. Ecol., 21, 844-853.

Prache S., Gordon I.J., Rook A.J., 1998 Foraging behaviour and diet selection in domestic herbivores. Ann. Zootech., 47, 335-345.

Prache S., Priolo A., Grolier P., 2003. Persistence of carotenoid pigments in the blood of concentrate-finished grazing sheep: Its significance for the traceability of grass-feeding. J. Anim. Sci., 81, 360-367.

Priolo A.,Vasta V., 2007. Effects of tannincontaining diets on small ruminant meat quality. It. J. Anim. Sci., 6, 527-530.

Rodrigues A., Andueza D., Picard F., Cecato U., Farruggia A., Baumont R., 2007. Valeur alimentaire et composition floristique des prairies permanentes : premiers résultats d'une étude conduite dans le Massif Central. Renc. Rech. Rum., 14, 241.

Roumet J.P., Fleury P., Jeannin B., 1996 Facteurs de variation et prévision de la production fourragère en zone de haute montagne. Fourrages, 145, 77-90.

Sanderson M A, Skinner R H, Barker D J Edwards G R, Tracy B F, Wedin D A., 2004. Plant species diversity and management of temperate forage and grazing land ecosystems. Crop Sci., 44, 1132-1144.

Sanderson M.A., Goslee S.C., Soder K.J., Skinner R.H., Tracy B.F., Deak A., 2007. Plant species diversity, ecosystem function, and pasture management - A perspective. Can. J. Plant Sci., 87, 479-487.

Sauvant D., Grolier P., Azais-Braesco V., 2002. Nutritional significance of vitamine A in dairy products. Encyclopaedia of Dairy Science, Academic Press, London., 2657-2664.

Scehovic J., 1990. Tannins et autres polymères polyphenoliques dans les plantes des prairies : détermination de leur teneur et de leur activité biologique. Rev. Suisse Agric., 22, -179.

Schreurs N.M., Marotti D.M., Tavendale M.H., Lane G.A., Barry T.N., Lopez-Villalobos N., McNabb W.C., 2007a. Concentration of 
indoles and other rumen metabolites in sheep after a meal of fresh white clover, perennial ryegrass or Lotus corniculatus and the appearance of indoles in the blood. J. Sci. Food Agric., 87, 1042-1051.

Schreurs N.M., Tavendale M.H., Lane G.A., Barry T.N., Lopez-Villalobos N., McNabb W.C., 2007b. Effect of different condensed tannin-containing forages, forage maturity and nitrogen fertiliser application on the formation of indole and skatole in vitro rumen fermentations. J. Sci. Food Agric., 87, 1076-1087.

Schreurs N.M., McNabb W.C., Tavendale M.H., Lane G.A., Barry T.N., Cummings T., Fraser K., Lopez-Villalobos N., Ramirez-Restrepo C.A., 2007c. Skatole and indole concentration of fat from lambs that had grazed perennial ryegrass/white clover pasture or Lotus corniculatus. Anim. Food Sci. Techn., 138, 254-271.

Schubiger F.X., Lehman J., Daccord R. Arrigo Y., Jeangros B., Scehovic J., 2001. Valeur nutritive des plantes des prairies. Digestibilité de la matière organique. Rev. Suisse Agric., 33, 275-279.

Selje N., Hoffmann E.M., Muetzel S., Ningrat R., Wallace R.J., Becker K., 2007. Results of a screening programme to identify plants or plant extracts that inhibit ruminal protein degradation. Br. J. Nutr., 98, 45-53.

Shaik,S.A., Terrill T.H., Miller J.E., Kouakou B., Kannan G., Kaplan R.M., Burke J.M., Mosjidis J.A., 2006. Sericea lespedeza hay as a natural deworming agent against gastrointestinal nematode infection in goats. Vet. Parasitol., 139, 150-157.

Sheath G.W., Coulon J.B., Young O.A., 2001. Grassland management and animal product quality. Grassland Ecosystems: an outlook into the $21^{\text {st }}$ century. $19^{\text {th }}$ Int. Grassl. Congr., Gomide J. A., Mattos W.R. S., Da Silva S.C. (Eds), Sao Paolo, Brazil, 1019-1026.
Soder K.J., Sanderson M.A., Stack J.L., Muller L.D., 2006. Intake performance of lactating cows grazing diverse forage mixtures. J. Dairy Sci., 89, 2158-2167.

Stahl W., Sies H., 2005. Bioactivity and protective effects of natural carotenoids. Biochimica et Biophysica Acta-Molecular Basis of Disease, 1740, 101-107.

Swift M.J., Izac A.M.N., van Noordwijk M., 2004. Biodiversity and ecosystem services in agricultural landscapes - are we asking the right questions? Agric. Ecosyst. Env., 104, 113-134.

Takayanagi R., Kato K.I., Ibayashi H., 1986. Relative inactivation of steroidogenic enzyme activities of in vitro vitamin E-depleted human adrenal microsomes by lipid peroxydation. Endocrinoly, 119, 464-470.

Tallowin J.R.B., Jefferson R.G., 1999. Hay production from lowland semi-natural grasslands: a review of implications for ruminant livestock systems. Grass Forage Sci., 54, 99-115.

Tornambe G., Cornu A., Verdier-Metz I., Pradel P., Kondjoyan N., Figueredo G., Hulin S., Bonanno A., Martin B., 2007a. Effet de l'addition dans le lait d'une huile essentielle de plantes de prairie naturelle sur les caractéristiques sensorielles de fromage de type Cantal. Renc. Rech. Rum., 14, 100.

Tornambe G., Ferlay A., Farruggia A., Chilliard Y., Loiseau P., Garel J.P., Martin B., 20007b. Effet de la diversité floristique des pâturages de montagne sur le profil en acides gras et les caractéristiques sensorielles des laits. Renc. Rech. Rum., 14, 333.

Trana A., Fedele V., Cifuni G.F., Impemba G., Claps S., Rubino R., 2005. Relationships among diet botanical composition, milk fatty acid and herbage fatty acid content in grazing goats. Options Méditerranéennes, Série A, Séminaires méditerranéens, FAO, 67, 269-273.
Urbach G., 1990. Effect of feed on flavor in dairy foods. J. Dairy Sci., 73, 3639-3650.

Verdier-Metz I., Coulon J.B., Pradel P., Viallon C., Albouy H., Berdagué J.L., 2000. Effect of the botanical composition of hay and casein genetic variants on the chemical and sensory characteristics of ripened Saint-Nectaire type cheeses. Lait, 80, 361-370.

Verdier-Metz I., Pradel P., Coulon J.B., 2002. Influence of the forage type and conservation on the cheese sensory properties. Multi-function grasslands. 19th Gen. Meet. Eur. Grassl Fed. 7, Durand, J. L., Emile, J. C., Huyghe, C., Lemaire, G. (Eds), La Rochelle, France, 604-605.

Vertuani A., Manfredi S., 2004. Antioxydants and pro-oxydants network: an overview. Curr. Pharmacol. Design., 10, 1677-1694.

Waller P.J., Ljungstrom B.L., Schwan O., Martin L.R., Morrison D.A., Rydzik A., 2006. Biological control of sheep parasites using Duddingtonia flagrans: Trials on commercial farms in Sweden. Acta Vet. Scand., 47, 23-32.

Whittington F.M., Dunn R., Nute G.R., Richardson R.I., Wood J.D., 2006. Effect of pasture type on lamb product quality. New developments in Sheepmeat quality. In: New Developments in Sheepmeat quality, Proc. Br. Soc. Anim. Sci., 27-31.

Young O.A., Cruickshank G.J., Maclean K.S., Muir P.D., 1994. Quality of Meat from Lambs Grazed on 7 Pasture Species in Hawkes Bay. N. Z. J. Agric. Res., 37, 177-186.

Young O.A., Lane G.A., Priolo A., Fraser K. 2003. Pastoral and species flavour in lambs raised on pasture, lucerne or maize. J. Sci. Food Agric., 83, 93-104.

Zeppa G., Giordano M., Gerbi V., Arlorio M., 2003. Fatty acid composition of Piedmont «Ossolano» cheese. Lait, 83, 167-173.

\section{Résumé}

Les prairies permanentes occupent environ un tiers de la surface agricole utile et présentent un grand potentiel de diversité biologique. L'élevage a de ce fait un rôle majeur à jouer dans la préservation de la biodiversité sur le territoire français. L'objectif de cette synthèse est d'aborder la question de la diversité floristique des prairies et de l'élevage en s'interrogeant sur l'intérêt de cette diversité construite par les éleveurs, pour les ruminants et les produits animaux. Une seule composante de la diversité biologique des prairies est prise en compte : la diversité floristique. Il est réalisé dans cette synthèse un état des connaissances pluridisciplinaires des effets de cette diversité sur la valeur nutritive des fourrages, l'ingestion des animaux, les caractéristiques sensorielles et nutritionnelles des produits laitiers et carnés, enfin sur deux aspects de la santé animale, la lutte contre les infestations par les strongles digestifs et la prévention des processus de peroxydation. Dans chacune des thématiques abordées, des effets liés à la composition botanique des prairies ont été mis en évidence, mais sans qu'il puisse être toujours fait la part entre les effets liés à la présence d'un grand nombre d'espèces dans la parcelle, les effets liés à la présence de certaines espèces et les effets liés au stade phénologique. Le rôle probable important des dicotylédones et notamment des légumineuses a été souligné à plusieurs reprises, en particulier du fait de leur plus grande teneur en métabolites secondaires que les graminées. Enfin, l'intérêt de fournir aux animaux des sources d'alimentation diversifiées a également été évoqué en particulier sur les aspects liés à l'ingestion et à la santé animale.

\section{Abstract}

\section{Is floristic diversity of permanent pastures important for ruminants and animal products?}

Semi natural grasslands covers about a third of the agricultural area used in France and presents a large biodiversity potential. Animal breeding consequently plays a key role in preserving the biodiversity of France. The aim of this synthesis is to examine the biodiversity as a result of the farmer's practices from the point of view of its value for ruminants and animals products. Only one aspect of biodiversity is included: floristic diversity. In this present paper, a state of multidisciplinary knowledge is realised about the effects of diversity on forage nutritive value, on animal ingestion, on sensory and nutritional traits of milk and meat products, and lastly on two different aspects of animal health: the prevention against the infestations by the gastrointestinal nematodes and the peroxydation process. With regard to 
this list above, the effects linked to botanical composition have been documented but we are not able to say if it is because of the large numbers of species or the presence of particular specie or the phenological state of the plants. The most likely role of dicotyledonous especially legumes has been underlined at various times in particular due to a larger concentration of secondary metabolites than herbs. Lastly, the interest of supplying animals with various feeds has been highlighted in particular on the aspects of ingestion and animal health.

FARRUGGIA A., MARTIN B., BAUMONT R., PRACHE S., DOREAU M., HOSTE H., DURAND D., 2008. Quels intérêts de la diversité floristique des prairies permanentes pour les ruminants et les produits animaux ? INRA Prod. Anim., 21, 181-200. 
